\title{
QED theory of elastic electron scattering on hydrogen-like ions involving formation and decay of autoionizing states
}

\author{
K. N. Lyashchenko $\odot,{ }^{1,2}$ D. M. Vasileva, ${ }^{1}$ O. Yu. Andreev $\odot,{ }^{1,3,}{ }^{*}$ and A. B. Voitkiv ${ }^{4}$ \\ ${ }^{1}$ Department of Physics, St. Petersburg State University, 7/9 Universitetskaya nab., St. Petersburg, 199034, Russia \\ ${ }^{2}$ Institute of Modern Physics, Chinese Academy of Sciences, Lanzhou 730000, China \\ ${ }^{3}$ Center for Advanced Studies, Peter the Great St. Petersburg Polytechnic University, 195251 St. Petersburg, Russia \\ ${ }^{4}$ Institute for Theoretical Physics I, Heinrich-Heine-University of Düsseldorf, Düsseldorf 40225, Germany
}

(Received 10 July 2019; published 28 January 2020)

\begin{abstract}
We develop ab initio relativistic QED theory for elastic electron scattering on hydrogen-like highly charged ions for impact energies where, in addition to direct (Coulomb) scattering, the process can also proceed via formation and consequent Auger decay of autoionizing states of the corresponding helium-like ions. Even so, the primary goal of the theory is to treat electron scattering on highly charged ions, a comparison with experiment shows that it can also be applied for relatively light ions covering thus a very broad range of the scattering systems. Using the theory we performed calculations for elastic electron scattering on $\mathrm{B}^{4+}, \mathrm{Ca}^{19+}, \mathrm{Fe}^{25+}, \mathrm{Kr}^{35+}$, and $\mathrm{Xe}^{53+}$. The theory was also generalized for collisions of hydrogen-like highly charged ions with atoms considering the latter as a source of (quasi)free electrons.
\end{abstract}

DOI: 10.1103/PhysRevResearch.2.013087

\section{INTRODUCTION}

Atomic systems with two or more electrons possess autoionizing states which can be highly visible in many processes studied by atomic physics, e.g., photo and impact ionization, dielectronic recombination, and photon or electron scattering. In particular, when an electron is incident on an ion, for certain (resonant) energies of the incident electron an autoionizing state can be formed. This state can then decay either via spontaneous radiative decay or via Auger decay due to the electron-electron interaction. In the former case dielectronic recombination takes place, whereas in the latter resonant electron scattering occurs. Depending on whether the initial and final ionic states coincide or not, the energy of the scattered electron can be equal to the energy of the incident electron (elastic resonant scattering) or differ from it (inelastic resonant scattering).

An incident electron can also scatter on an ion without excitation of the internal degrees of freedom of the ion. In such a case, scattering proceeds via the Coulomb force acting between the electron and the (partially screened) nucleus of the ion. This scattering channel is nonresonant and elastic and its amplitude should be added coherently to the amplitude for the elastic resonant scattering. As a result, there appears interference between these two channels which has to be taken into account in a proper treatment of elastic scattering.

\footnotetext{
*o.y.andreev@spbu.ru

Published by the American Physical Society under the terms of the Creative Commons Attribution 4.0 International license. Further distribution of this work must maintain attribution to the author(s) and the published article's title, journal citation, and DOI.
}

Resonant scattering was extensively studied for nonrelativistic electrons incident on light ions [for instance, $\left.e^{-}+\mathrm{He}^{+}(1 s) \rightarrow \mathrm{He}^{* *} \rightarrow \mathrm{He}^{+}(1 s)+e^{-}\right]$. This scattering becomes especially important at large scattering angles (as viewed in the rest frame of the ion) where the contribution of the potential Coulomb scattering is minimal. Therefore, experimental investigations (which often replace electron-ion collisions by ion-atom collisions in which atomic electrons are regarded as quasifree) were mainly focused on such angles (see, e.g., [1-6]). There exist also a large number of calculations for resonant electron-ion scattering in which a nonrelativistic electron interacts with a light ion (see, e.g., [5-8]).

In sharp contrast, the studies on resonant scattering of an electron on a highly charged ion, in which relativistic and QED effects can become of importance, are almost absent with no experimental data and merely two theoretical papers [9,10]. In [9] scattering of an electron on a hydrogen-like uranium ion was considered, however, only the resonant part of the scattering was calculated, whereas the potential Coulomb part as well as the interference between them were not taken into account. In [10] resonant electron scattering on helium-like ions with atomic numbers $60 \leqslant Z \leqslant 92$ proceeding via the formation of autoionizing states $\left[\left(1 s 2 p_{1 / 2}\right) n l\right]_{1 / 2}$ and $[(1 s 2 s) n l]_{1 / 2}$ with the principal quantum numbers $5 \leqslant$ $n \leqslant 7$ is presented. The goal of [10] was to investigate the possibility of experimental study of the parity nonconservation effects in this process. We note that for the heavy ions the potential Coulomb part is much larger than the resonant part (particularly, this concerns the contribution of highly excited states), so that the contribution of the resonant part including its interference with the Coulomb part is negligible.

In case of scattering on highly charged ions, the electrons are subjected to a very strong field generated by the ionic nucleus. As a result, the account of relativistic and QED effects 
may become of great importance for a proper description of the scattering process.

In this paper we consider elastic electron scattering on hydrogen-like highly charged ions for impact energies where the presence of autoionizing states of the corresponding helium-like ions can actively influence this process. To this end, we shall develop ab initio relativistic QED theory of this process, which enables one to address both its resonant and Coulomb parts in a unified and self-consistent way. Relativistic units are used throughout unless otherwise is stated.

\section{GENERAL THEORY}

We consider elastic electron scattering on a hydrogen-like ion which is initially in its ground state,

$$
e_{\boldsymbol{p}_{i}, \mu_{i}}^{-}+X^{(Z-1)+}(1 s) \rightarrow e_{p_{f}, \mu_{f}}^{-}+X^{(Z-1)+}(1 s),
$$

where $Z$ is the atomic number of the ion $X$. If the energy of the initial state of the electron system, which consists of the incident electron $e_{\boldsymbol{p}, \mu}^{-}$with an asymptotic momentum $\boldsymbol{p}$ and polarization $\mu$ and the $1 s$ electron bound in the ion, is close to the energy of a doubly excited (autoionizing) state of the corresponding helium-like ion, the resonant scattering channel

$$
\begin{aligned}
e_{\boldsymbol{p}_{i}, \mu_{i}}^{-}+X^{(Z-1)+}(1 s) & \rightarrow X^{(Z-2)+}(d) \\
& \rightarrow e_{\boldsymbol{p}_{f}, \mu_{f}}^{-}+X^{(Z-1)+}(1 s),
\end{aligned}
$$

where $d$ is a doubly excited state, becomes of importance. Here, the scattering proceeds via the formation of a doubly excited state $(d)$ and its subsequent Auger decay. This channel is driven by the interelectron interaction.

One can expect that in case of highly charged ions the main channel of the electron-ion scattering process (1) is Coulomb scattering, which is nonresonant. Therefore, in order to investigate the resonant structure experimentally, the electron scattering to very large angles $\left(\simeq 180^{\circ}\right)$, for which the contribution of the main channel is minimal, should be considered [5]. Accordingly, one needs to calculate the differential cross section.

We shall consider the scattering process (1) using the Furry picture [11], in which the action of a strong external field (for example, the field of the ionic nucleus) on the electrons is taken into account from the very beginning. The electrons interact with each other via the interaction with the quantized electromagnetic and electron-positron fields, which is accounted for by using perturbation theory.

The ingoing $(+)$ and outgoing (-) wave functions of an electron in an external central electric field with a asymptotic momentum $(\boldsymbol{p})$ and polarization $(\mu)$ can be presented as [12]

$$
\psi_{\boldsymbol{p} \mu}^{( \pm)}(\boldsymbol{r})=N \sum_{j l m} \Omega_{j l m}^{+}(\boldsymbol{v}) v_{\mu}(\boldsymbol{v}) e^{ \pm i \phi_{j l} l} i^{l} \psi_{\varepsilon j l m}(\boldsymbol{r}),
$$

where

$$
N=\frac{(2 \pi)^{3 / 2}}{\sqrt{p \varepsilon}}
$$

is the normalization factor, $\boldsymbol{v}=\boldsymbol{p} /|\boldsymbol{p}|$ is the unit vector defining the angular dependence of the momentum $\boldsymbol{p}$. The wave functions $\psi_{\varepsilon j l m}(\boldsymbol{r})$ describe electrons with the energy $\varepsilon=\sqrt{1+p^{2}}$, the total angular momentum $j$, its projection $m$ and parity defined by the orbital momentum $l$, and $\phi_{j l}$ are the phases determined by the external field [see Eq. (B1) in Appendix B]. These wave functions are normalized according to

$$
\int d \boldsymbol{r} \psi_{\varepsilon^{\prime} j^{\prime} l^{\prime} m^{\prime}}^{+}(\boldsymbol{r}) \psi_{\varepsilon j l m}(\boldsymbol{r})=\delta\left(\varepsilon^{\prime}-\varepsilon\right) \delta_{j^{\prime} j} \delta_{l^{\prime} l} \delta_{m^{\prime} m},
$$

where $\delta$ denotes either the delta function or the Kronecker symbol, respectively. The spherical bispinor reads as [13]

$$
\Omega_{j l m}(\boldsymbol{v})=\sum_{m_{l} m_{s}} C_{j m}^{l s}\left(m_{l} m_{s}\right) Y_{l m_{l}}(\boldsymbol{v}) \chi_{m_{s}},
$$

where $C_{j m}^{l s}\left(m_{l} m_{s}\right)=\left\langle l m_{l} s m_{s} \mid j m\right\rangle$ are the Clebsch-Gordan coefficients, $Y_{l m_{l}}(\boldsymbol{v})$ are spherical harmonics, and $\chi_{m_{s}}$,

$$
\chi_{+1 / 2}=\left(\begin{array}{l}
1 \\
0
\end{array}\right), \quad \chi_{-1 / 2}=\left(\begin{array}{l}
0 \\
1
\end{array}\right)
$$

are spinors. Further, in (3) there are also spinors $v_{\mu}(\boldsymbol{v})$, which are defined according to

$$
\begin{gathered}
\frac{1}{2} \boldsymbol{v} \boldsymbol{\sigma} v_{\mu}(\boldsymbol{v})=\mu v_{\mu}(\boldsymbol{v}), \\
\left(v_{\mu^{\prime}}(\boldsymbol{v}), v_{\mu}(\boldsymbol{v})\right)=\delta_{\mu^{\prime} \mu}, \\
v_{\mu}\left(\boldsymbol{e}_{z}\right)=\chi_{\mu},
\end{gathered}
$$

where $\boldsymbol{\sigma}$ is the Pauli vector, $\boldsymbol{e}_{z}$ is the unit vector along the $z$ axis. Then, the wave functions $\psi_{\boldsymbol{p} \mu}^{( \pm)}(\boldsymbol{r})$ are normalized as

$$
\begin{aligned}
\int d \boldsymbol{r} \psi_{\boldsymbol{p}^{\prime} \mu^{\prime}}^{( \pm)+}(\boldsymbol{r}) \psi_{\boldsymbol{p} \mu}^{( \pm)}(\boldsymbol{r})= & (2 \pi)^{3} \delta\left(\boldsymbol{p}^{\prime}-\boldsymbol{p}\right) \delta_{\mu^{\prime} \mu} \\
= & \frac{(2 \pi)^{3}}{\varepsilon p} \delta\left(\varepsilon^{\prime}-\varepsilon\right) \delta\left(\cos \theta^{\prime}-\cos \theta\right) \\
& \times \delta\left(\varphi^{\prime}-\varphi\right) \delta_{\mu^{\prime} \mu} .
\end{aligned}
$$

For the process of elastic electron scattering (1), the contribution of its Coulomb part to the amplitude is very important. The Coulomb scattering amplitude is usually calculated by studying the asymptotics of the electron wave functions in the Coulomb field [12].

The resonant part of the scattering process (2) is due to the formation and decay of autoionizing states of the corresponding helium-like ion. In this paper, for the description of autoionizing states within the QED theory the line-profile approach (LPA) will be employed [14], where the QED perturbation theory is used. In particular, it is necessary to take into account various corrections such as the interelectron interaction corrections and the relativistic corrections to the scattering amplitude.

In order to make the consideration of both Coulomb and resonant parts of the scattering amplitude self-consistent, we shall apply the formal theory of scattering for the Coulomb potential considering it by using perturbation theory.

\section{A. Coulomb scattering amplitude}

For simplicity, in this section we limit ourselves to the consideration of a one-electron system. Within the scattering theory developed in $[15,16]$, the in $(+)$ and out $(-)$ states 
satisfy the Lippmann-Schwinger equation

$$
\psi_{\boldsymbol{p} \mu}^{( \pm)}(\boldsymbol{r})=\phi_{\boldsymbol{p} \mu}(\boldsymbol{r})+\frac{1}{\varepsilon-\hat{H}_{0} \pm i 0} \hat{V} \psi_{\boldsymbol{p} \mu}^{( \pm)}(\boldsymbol{r}),
$$

where $\hat{V}$ represents the scattering potential. Here, the function $\phi_{\boldsymbol{p} \mu}(\boldsymbol{r})$ describes a free electron and can be obtained from Eq. (3) by setting $Z=0$ :

$$
\begin{gathered}
\phi_{\boldsymbol{p} \mu}(\boldsymbol{r})=u_{\mu}(p) e^{i \boldsymbol{p r}} \\
u_{\mu}(p)=\frac{1}{\sqrt{2 \varepsilon}}\left(\begin{array}{c}
\sqrt{\varepsilon+1} v_{\mu}(\boldsymbol{v}) \\
\sqrt{\varepsilon-1}(\boldsymbol{\sigma} \boldsymbol{v}) v_{\mu}(\boldsymbol{v})
\end{array}\right) .
\end{gathered}
$$

Following [15] we introduce the $R$-matrix elements according to

$$
\begin{aligned}
R_{i f} & =\left\langle\phi_{\boldsymbol{p}_{f} \mu_{f}}|\hat{V}| \psi_{\boldsymbol{p}_{i} \mu_{i}}^{(+)}\right\rangle \\
& =\left\langle\phi_{\boldsymbol{p}_{f} \mu_{f}}|\hat{V}| \phi_{\boldsymbol{p}_{i} \mu_{i}}\right\rangle+\left\langle\phi_{\boldsymbol{p}_{f} \mu_{f}}\left|\hat{V} \frac{1}{\varepsilon_{i}-\hat{H}_{0}+i 0} \hat{V}\right| \psi_{\boldsymbol{p}_{i} \mu_{i}}^{(+)}\right\rangle
\end{aligned}
$$

The $S$-matrix elements are related to the $R$ matrix as follows:

$$
S_{i f}=\left\langle\phi_{p_{f} \mu_{f}}|\hat{S}| \phi_{p_{i} \mu_{i}}\right\rangle=\delta_{i f}-2 \pi i \delta\left(\varepsilon_{i}-\varepsilon_{f}\right) R_{i f} .
$$

Since the $S$-matrix elements can be also written as

$$
S_{i f}=\left\langle\psi_{\boldsymbol{p}_{f} \mu_{f}}^{(-)} \mid \psi_{\boldsymbol{p}_{i} \mu_{i}}^{(+)}\right\rangle
$$

we obtain that $(f \neq i)$

$$
\left\langle\psi_{\boldsymbol{p}_{f} \mu_{f}}^{(-)} \mid \psi_{\boldsymbol{p}_{i} \mu_{i}}^{(+)}\right\rangle=(-2 \pi i) \delta\left(\varepsilon_{f}-\varepsilon_{i}\right) R_{i f} .
$$

In the case of Coulomb scattering, $\hat{V}=-\alpha Z / r$, the states $\psi_{\boldsymbol{p}_{i} \mu_{i}}^{( \pm)}$can be conveniently evaluated using the expansions (3), which enables us to obtain the following expression:

$$
R_{i f}^{\text {Coul }}=N^{2} \frac{(-1) p}{(2 \pi)^{2}} \sum_{m}\left(v_{\mu_{f}}\left(\boldsymbol{v}_{f}\right)\right)_{m}^{*} M_{m \mu_{i}}(\theta, \varphi)
$$

(a detailed derivation of $R^{\text {Coul }}$ is presented in Appendix A). Following [17] we introduced the matrix $M$ :

$$
\begin{aligned}
& M_{+1 / 2,+1 / 2}(\theta, \varphi)=f(\theta), \quad M_{+1 / 2,-1 / 2}(\theta, \varphi)=g(\theta) e^{-i \varphi}, \\
& M_{-1 / 2,+1 / 2}(\theta, \varphi)=-g(\theta) e^{i \varphi}, \quad M_{-1 / 2,-1 / 2}(\theta, \varphi)=f(\theta),
\end{aligned}
$$

where $f(\theta)$ and $g(\theta)$ are the relativistic scattering amplitudes

$$
\begin{gathered}
f(\theta)=\frac{1}{2 \pi i} \sum_{j l}|\varkappa|\left(e^{2 i \phi_{\varkappa}}-1\right) P_{l}(\cos \theta), \\
g(\theta)=\frac{1}{2 \pi i} \sum_{l}\left(e^{2 i \phi_{\varkappa=-l-1}}-e^{2 i \phi_{\varkappa=l}}\right) P_{l}^{1}(\cos \theta) .
\end{gathered}
$$

\section{B. Coulomb scattering amplitude within the LPA}

In the QED theory the scattering matrix $(S)$ can be represented as the sum of the normal ordered products of field operators corresponding to different processes of particle scattering. Each normal ordered product and, consequently, any scattering process can be represented graphically according to the Feynman rules. In the corresponding matrix elements, the integration over $x^{\mu}=(t, \boldsymbol{r}) 4$-vectors is performed. If in the process the energy is conserved, after the integration over the

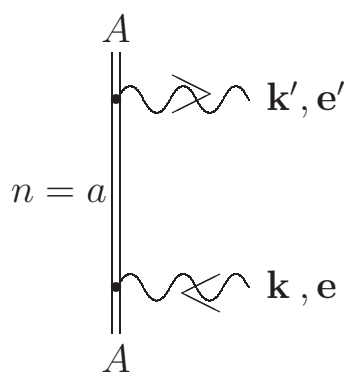

FIG. 1. Feynman graph describing the elastic photon scattering on an atomic electron. The double solid line denotes the electron in the external potential $V^{\mathrm{F}}$ (the Furry picture). The wavy lines with the arrows describe the emission and absorption of a photon with momentum $\boldsymbol{k}$ and polarization $e$.

time variables the matrix element can be written as [12]

$$
S_{i f}^{\mathrm{QED}}=(-2 \pi i) \delta\left(\varepsilon_{f}-\varepsilon_{i}\right) U_{i f}^{\mathrm{QED}},
$$

where $U_{i f}^{\mathrm{QED}}$ is the amplitude. In QED of strong fields the $S$-matrix elements and, correspondingly, the amplitude $U_{i f}$ are evaluated with the use of perturbation theory [12]. For the description of highly charged ions within QED theory, special methods are employed. Most of QED calculations were performed using the adiabatic $S$-matrix approach [18-20], the two-time Green's function method [21], the covariantevolution-operator method [22], and the LPA [14]. In this paper we employ the LPA.

We shall now establish the relationship between the amplitude $R_{i f}^{\text {Coul }}$ defined by Eq. (15) and the corresponding amplitudes derived within the LPA. Let us evaluate the scattering amplitude corresponding to the interaction with an external field $V^{\text {ext }}$ in the one-electron case. Within the LPA the initial and final states (the reference states) are described as resonances in the process of scattering. As an auxiliary process, it is normally most convenient to take elastic photon scattering. For the properties of the reference states to be independent of the details of the scattering process, the resonance approximation is employed [14]. In this approximation, the line profile is interpolated by the Lorentz contour, the position of the resonance and its width define the energy and width of the corresponding state.

We consider the process of elastic photon scattering on one-electron ion initially being in its ground state. The Feynman graphs corresponding to this process in the zeroth order of the perturbation theory are depicted in Fig. 1. The corresponding $S$-matrix element reads as $[12,14]$

$$
\begin{aligned}
S_{i f}^{\mathrm{QED}}= & (-i e)^{2} \int d^{4} x_{u} d^{4} x_{d} \bar{\psi}_{u}\left(x_{u}\right) \gamma^{\mu_{u}} S\left(x_{u}, x_{d}\right) \gamma^{\mu_{d}} \psi_{d}\left(x_{d}\right) \\
& \times A_{\mu_{u}}^{*\left(k^{\prime}, \lambda^{\prime}\right)}\left(x_{u}\right) A_{\mu_{d}}^{(k, \lambda)}\left(x_{d}\right) .
\end{aligned}
$$

We assume that $\psi_{u}=\psi_{d}$ describes the $1 s$ electron. The electron propagator $S\left(x_{u}, x_{d}\right)$ can be written as

$$
S\left(x_{u}, x_{d}\right)=\frac{i}{2 \pi} \int d \omega_{1} e^{-i \omega_{1}\left(t_{u}-t_{d}\right)} \sum_{n} \frac{\psi_{n}\left(\boldsymbol{r}_{u}\right) \bar{\psi}_{n}\left(\boldsymbol{r}_{d}\right)}{\omega_{1}-\varepsilon_{n}(1-i 0)},
$$

where the sum runs over the entire Dirac spectrum. Further, $A_{\mu_{d}}^{(k, \lambda)}\left(x_{d}\right)$ and $A_{\mu_{u}}^{*\left(k^{\prime}, \lambda^{\prime}\right)}\left(x_{u}\right)$ refer to the absorbed and emitted 


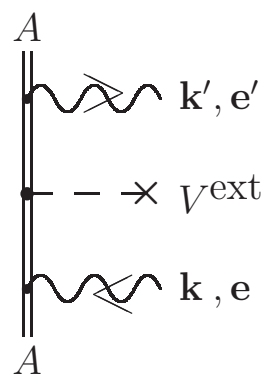

FIG. 2. Feynman graph corresponding to the insertion describing the interaction with the external field $V^{\text {ext }}$. The dotted line with a cross denotes the interaction with the field $V^{\text {ext }}$. The notations are the same as in Fig. 1.

photons, respectively, $k^{\mu}=(\omega, \boldsymbol{k})$ is the 4-vector of photon momentum, $\lambda$ describes the photon polarization. By inserting (25) into Eq. (24) and integrating over the time and frequency variables $t_{u}, t_{d}$, and $\omega_{n}$ we obtain

$$
S_{i f}^{\mathrm{QED}}=(-2 \pi i) \delta\left(\omega^{\prime}+\varepsilon_{u}-\omega-\varepsilon_{d}\right) e^{2} \sum_{n} \frac{A_{u n}^{*\left(k^{\prime}, \lambda^{\prime}\right)} A_{n d}^{(k, \lambda)}}{\omega+\varepsilon_{d}-\varepsilon_{n}},
$$

where one-electron matrix elements

$$
\begin{aligned}
A_{n d}^{(k, \lambda)} & =\int d^{3} \boldsymbol{r} \bar{\psi}_{n}(\boldsymbol{r}) \gamma^{\mu} A_{\mu}^{(k, \lambda)}(\boldsymbol{r}) \psi_{d}(\boldsymbol{r}), \\
A_{u n}^{*\left(k^{\prime}, \lambda^{\prime}\right)} & =\int d^{3} \boldsymbol{r} \bar{\psi}_{u}(\boldsymbol{r}) \gamma^{\mu} A_{\mu}^{*\left(k^{\prime}, \lambda^{\prime}\right)}(\boldsymbol{r}) \psi_{n}(\boldsymbol{r})
\end{aligned}
$$

were introduced.

In the first order of the perturbation theory we consider one interaction with an external field $A_{\mu}^{\text {ext }}(x)=\left(V^{\text {ext }}(\boldsymbol{r}), \mathbf{0}\right)$. The Feynman graph describing this interaction is presented in Fig. 2. The corresponding $S$-matrix element reads as

$$
\begin{aligned}
S_{i f}^{\mathrm{QED}(1)}= & (-i e)^{3} \int d^{4} x_{u} d^{4} x_{d} d^{4} x \bar{\psi}_{u}\left(x_{u}\right) \gamma^{\mu_{u}} A_{\mu_{u}}^{*\left(k^{\prime}, \lambda^{\prime}\right)}\left(x_{u}\right) \\
& \times S\left(x_{u}, x\right) \gamma^{\mu} A_{\mu}^{\mathrm{ext}}(x) S\left(x, x_{d}\right) A_{\mu_{d}}^{(k, \lambda)}\left(x_{d}\right) \gamma^{\mu_{d}} \psi_{d}\left(x_{d}\right) .
\end{aligned}
$$

The integration over the time and frequency variables leads to

$$
\begin{aligned}
S_{i f}^{\mathrm{QED}(1)}= & (-2 \pi i) \delta\left(\omega^{\prime}+\varepsilon_{u}-\omega-\varepsilon_{d}\right) e^{2} \sum_{n_{1} n_{2}} \frac{A_{u n_{1}}^{*\left(k^{\prime}, \lambda^{\prime}\right)}}{\left(\omega^{\prime}+\varepsilon_{u}-\varepsilon_{n_{1}}\right)} \\
& \times V_{n_{1} n_{2}}^{\operatorname{ext}} \frac{A_{n_{2} d}^{(k, \lambda)}}{\left(\omega+\varepsilon_{d}-\varepsilon_{n_{2}}\right)},
\end{aligned}
$$

where

$$
V_{n_{1} n_{2}}^{\mathrm{ext}}=e \int d^{3} \boldsymbol{r} \psi_{n_{1}}^{+}(\boldsymbol{r}) V^{\mathrm{ext}}(\boldsymbol{r}) \psi_{n_{2}}(\boldsymbol{r})
$$

Within the LPA the reference states (the initial and final states) are defined via resonances in the process of elastic photon scattering. Accordingly, we are interested in (we note that $\varepsilon_{u}=\varepsilon_{d}$ )

$$
\begin{gathered}
\omega^{\prime}+\varepsilon_{u} \approx \varepsilon_{f}, \\
\omega+\varepsilon_{d} \approx \varepsilon_{i} .
\end{gathered}
$$

In the resonance approximation, the reference states are described in such a way that their properties do not depend on a specific scattering process. Accordingly, retaining only terms with $n_{1}=f, n_{2}=i$, in the resonance approximation we get

$$
\begin{aligned}
S_{i f}^{\mathrm{QED}(1) \mathrm{res}}= & (-2 \pi i) \delta\left(\omega^{\prime}+\varepsilon_{u}-\omega-\varepsilon_{d}\right) e^{2} \frac{A_{u f}^{*\left(k^{\prime}, \lambda^{\prime}\right)}}{\left(\omega^{\prime}+\varepsilon_{u}-\varepsilon_{f}\right)} \\
& \times V_{f i}^{\mathrm{ext}} \frac{A_{i d}^{(k, \lambda)}}{\left(\omega+\varepsilon_{d}-\varepsilon_{i}\right)} .
\end{aligned}
$$

The second order of the perturbation theory with respect to the interaction with the external field reads as

$$
\begin{aligned}
S_{i f}^{\mathrm{QED}(2)}= & (-2 \pi i) \delta\left(\omega^{\prime}+\varepsilon_{u}-\omega-\varepsilon_{d}\right) e^{2} \\
& \times \sum_{n_{1} n_{2} n_{3}} \frac{A_{u n_{1}}^{*\left(k^{\prime}, \lambda^{\prime}\right)}}{\left(\omega^{\prime}+\varepsilon_{u}-\varepsilon_{n_{1}}\right)} V_{n_{1} n_{2}}^{\mathrm{ext}} \frac{1}{\left(\omega+\varepsilon_{d}-\varepsilon_{n_{2}}\right)} \\
& \times V_{n_{2} n_{3}}^{\mathrm{ext}} \frac{A_{n_{3} d}^{(k, \lambda)}}{\left(\omega+\varepsilon_{d}-\varepsilon_{n_{3}}\right)} .
\end{aligned}
$$

In the resonance approximation we keep only the term with $n_{2}=n_{3}=i$ :

$$
\begin{aligned}
S_{i f}^{\mathrm{QED}(2) \mathrm{res}}= & (-2 \pi i) \delta\left(\omega^{\prime}+\varepsilon_{u}-\omega-\varepsilon_{d}\right) e^{2} \frac{A_{u f}^{*\left(k^{\prime}, \lambda^{\prime}\right)}}{\left(\omega^{\prime}+\varepsilon_{u}-\varepsilon_{f}\right)} \\
& \times V_{f i}^{\mathrm{ext}} \frac{1}{\left(\omega+\varepsilon_{d}-\varepsilon_{i}\right)} V_{i i}^{\mathrm{ext}} \frac{A_{i d}^{(k, \lambda)}}{\left(\omega+\varepsilon_{d}-\varepsilon_{i}\right)} .
\end{aligned}
$$

The third order of perturbation theory in the resonance approximation reads as

$$
\begin{aligned}
S_{i f}^{\mathrm{QED}(3) \mathrm{res}}= & (-2 \pi i) \delta\left(\omega^{\prime}+\varepsilon_{u}-\omega-\varepsilon_{d}\right) \\
& \times e^{2} \sum_{n_{1} n_{2}} \frac{A_{u f}^{*\left(k^{\prime}, \lambda^{\prime}\right)}}{\left(\omega^{\prime}+\varepsilon_{u}-\varepsilon_{f}\right)} V_{f i}^{\mathrm{ext}} \frac{1}{\left(\omega+\varepsilon_{d}-\varepsilon_{i}\right)} \\
& \times V_{i i}^{\mathrm{ext}} \frac{1}{\left(\omega+\varepsilon_{d}-\varepsilon_{i}\right)} V_{i i}^{\mathrm{ext}} \frac{A_{i d}^{(k, \lambda)}}{\left(\omega+\varepsilon_{d}-\varepsilon_{i}\right)}
\end{aligned}
$$

In the resonance approximation the series of the perturbation theory composes a geometric progression

$$
\begin{aligned}
\sum_{l=0}^{\infty} S_{i f}^{\mathrm{QED}(l) \mathrm{res}} & =(-2 \pi i) \delta\left(\omega^{\prime}+\varepsilon_{u}-\omega-\varepsilon_{d}\right) e^{2} \sum_{l=0}^{\infty} \frac{A_{u f}^{*\left(k^{\prime}, \lambda^{\prime}\right)}}{\left(\omega^{\prime}+\varepsilon_{u}-\varepsilon_{f}\right)} V_{f i}^{\mathrm{ext}}\left[\frac{1}{\left(\omega+\varepsilon_{d}-\varepsilon_{i}\right)} V_{i i}^{\mathrm{ext}}\right]^{l} \frac{A_{i d}^{(k, \lambda)}}{\left(\omega+\varepsilon_{d}-\varepsilon_{i}\right)} \\
& =(-2 \pi i) \delta\left(\omega^{\prime}+\varepsilon_{u}-\omega-\varepsilon_{d}\right) e^{2} \frac{A_{u f}^{*\left(k^{\prime}, \lambda^{\prime}\right)}}{\left(\omega^{\prime}+\varepsilon_{u}-\varepsilon_{f}\right)} V_{f i}^{\mathrm{ext}} \frac{A_{i d}^{(k, \lambda)}}{\left(\omega+\varepsilon_{d}-\varepsilon_{i}-V_{i i}^{\mathrm{ext}}\right)} .
\end{aligned}
$$


Thus, the summation of the infinite series resulted in a shift of the position of the resonance, which represents a correction to the energy of the reference state. This procedure was first applied in [23] for the derivation of the natural line shape within the QED theory, where the correction due to the electron self-energy was considered. We also note that the corrections to the photon scattering amplitude for a few electron ions were discussed in [14].

Within the LPA we introduce the amplitude

$$
U_{i f}=\sum_{l=1}^{\infty} U_{i f}^{(l)},
$$

where

$$
\begin{gathered}
U_{i f}^{(1)}=V_{f i}^{\mathrm{ext}}, \\
U_{i f}^{(2)}=\sum_{n} V_{f n}^{\mathrm{ext}} \frac{1}{\left(\omega+\varepsilon_{d}-\varepsilon_{n}\right)} V_{n i}^{\mathrm{ext}}, \\
U_{i f}^{(3)}=\sum_{n_{1} n_{2}} V_{f n_{1}}^{\mathrm{ext}} \frac{1}{\left(\omega+\varepsilon_{d}-\varepsilon_{n_{1}}\right)} V_{n_{1} n_{2}}^{\mathrm{ext}} \frac{1}{\left(\omega+\varepsilon_{d}-\varepsilon_{n_{2}}\right)} V_{n_{2} i}^{\mathrm{ext}} .
\end{gathered}
$$

In the resonance approximation we can set $\omega+\varepsilon_{d}=\varepsilon_{i}$. The amplitude (39) describes the transition $(i \rightarrow f)$ caused by the external field $V^{\text {ext }}$.

Introducing the operator $\Delta \hat{V}$, which is defined by its matrix elements as

$$
\Delta V_{n_{1} n_{2}}=V_{n_{1} n_{2}}^{\text {ext }},
$$

the amplitude can be rewritten as

$$
\begin{aligned}
U_{i f}= & \left\langle\Psi_{f}^{(0)}|\Delta \hat{V}| \Psi_{i}^{(0)}\right\rangle+\left\langle\Psi_{f}^{(0)}\left|\Delta \hat{V} \frac{1}{\varepsilon_{i}-\hat{H}_{0}+i 0} \Delta \hat{V}\right| \Psi_{i}^{(0)}\right\rangle \\
& +\left\langle\Psi_{f}^{(0)}\left|\Delta \hat{V} \frac{1}{\varepsilon_{i}-\hat{H}_{0}+i 0} \Delta \hat{V} \frac{1}{\varepsilon_{i}-\hat{H}_{0}+i 0} \Delta \hat{V}\right| \Psi_{i}^{(0)}\right\rangle \\
& +\cdots \\
= & \sum_{l=0}^{\infty}\left\langle\Psi_{f}^{(0)}\left|\Delta \hat{V}\left[\frac{1}{\varepsilon_{i}-\hat{H}_{0}+i 0} \Delta \hat{V}\right]^{l}\right| \Psi_{i}^{(0)}\right\rangle
\end{aligned}
$$

The one-electron wave functions $\Psi_{n}^{(0)}$ describe electrons noninteracting with the external field $V_{n_{1} n_{2}}^{\text {ext }}$ :

$$
\hat{H}_{0} \Psi_{n}^{(0)}=\varepsilon_{n} \Psi_{n}^{(0)} .
$$

Here, $\hat{H}_{0}$ is the Dirac Hamiltonian

$$
\hat{H}_{0}=\boldsymbol{\alpha} \hat{\boldsymbol{p}}+\beta m+V^{\mathrm{F}}(\boldsymbol{r}),
$$

where $\alpha$ and $\beta$ are the Dirac matrices and the choice of the potential $V^{\mathrm{F}}$ defines the Furry picture employed.

The amplitude $U_{i f}$ in Eq. (44) can be also written as

$$
U_{i f}=\left\langle\Psi_{f}^{(0)}|\Delta \hat{V}| \Phi_{i}\right\rangle,
$$

where

$$
\Phi_{i}=\Psi_{i}^{(0)}+\frac{1}{\varepsilon_{i}-\hat{H}_{0}+i 0} \Delta \hat{V} \Phi_{i} .
$$

The function $\Phi_{i}$ is a solution of the following equation:

$$
\left(\hat{H}_{0}+\Delta \hat{V}\right) \Phi_{i}=\varepsilon_{i} \Phi_{i} .
$$

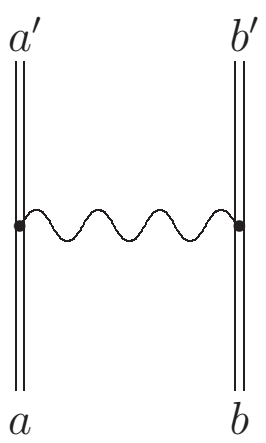

FIG. 3. Feynman graph corresponding to the first-order interelectron interaction in two-electron ion (one-photon exchange graph).

We note that the amplitude (47), in which the exact state is given by (48), essentially coincides with the amplitude (15), where the exact state is given by (12). Therefore, we conclude that the amplitude $U_{i f}$ obtained within the LPA, a QED approach, coincides with the amplitude $R_{i f}$, which follows from (relativistic) quantum mechanics and which, in particular, can be evaluated using Eq. (18). One should add that in the framework of the LPA also QED corrections, such as electron self-energy, vacuum polarization, photon exchange corrections, can be taken into account using the procedure described above. Thus, in general, the operator $\Delta \hat{V}$ includes also the corresponding QED corrections.

\section{Implementation of the LPA for the description of elastic resonant scattering}

The scattering amplitude corresponding to the interelectron interaction is given by Feynman graphs depicted in Figs. 3 and 4. The graph in Fig. 3 represents the one-photon exchange correction, the graphs in Figs. 4(a) and 4(b) refer to the two-photon exchange corrections; the three- and more-photon exchange corrections could also be considered. One should note, however, that in the process under consideration all these graphs need a special treatment because they contain divergences.

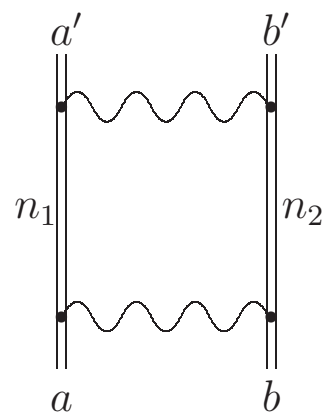

(a)

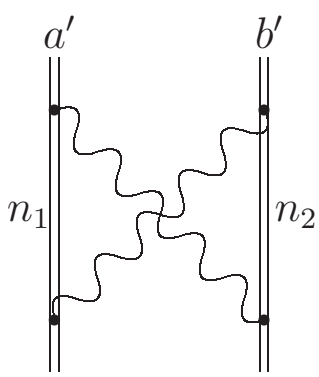

(b)
FIG. 4. Feynman graphs corresponding to the second-order interelectron interaction in two-electron ion (two-photon exchange graphs). 
For the description of processes, which involve electrons interacting with the field of a highly charged nucleus, the Furry picture is normally used, in which the interaction of the electrons with the potential of the nucleus $V^{\mathrm{F}}=-\alpha Z / r$ is fully taken into account from the onset, whereas the interelectron interaction is considered using a QED perturbation theory. However, in the case of elastic scattering such an approach leads to divergent results arising from the application of the perturbation theory to a long-range Coulomb interaction between bound and free electrons.

In order to modify the "standard" approach, we note that the incident and scattered electron most of the time is moving in the Coulomb field of a hydrogen-like ion with the net charge $Z-1$. Therefore, we shall avoid the use of the perturbation theory for the interaction of the free electron with the bound electron by employing the Furry picture in which the free electron is supposed to move in the field of the nuclear charge $Z-1$. Then, the interaction of the free electron with the bound electron together with its interaction with the "remaining" charge of the nucleus $[Z-(Z-1)=1]$ is already a short-range interaction since it corresponds to the interaction with an electrically neutral system [the charge of the bound electron $(e)$ smeared out over the size of its bound state and the "remaining" charge of the nucleus $(-e)$ ]. Now, the divergences do not arise and this short-range interaction can be accounted for by using the perturbation theory.

Thus, our consideration involves the following points. (i) The wave functions of the incident and scattered electron are obtained by solving the Dirac equation with the potential $-\alpha(Z-1) / r$. (ii) The wave functions of all other electrons (bound and virtual) are derived from the Dirac equation with the potential $-\alpha Z / r$. (iii) The interaction of the continuum electrons with the "remaining" charge of the nucleus is calculated with the use of perturbation theory. (iv) The interelectron interaction is considered as the interaction with the quantized electromagnetic and electron-positron fields within the QED perturbation theory. (v) The divergences arising from the longrange Coulomb interaction of the continuum electron with the remaining charge of the nucleus and with the bound electron can be regularized and cancel each other.

The amplitude of the Coulomb scattering given by Eq. (19) formally corresponds to taking into account the Feynman graphs depicted in Fig. 5, where graphs (a) and (b) represent the first and second terms in the right side of Eq. (15). For the derivation of the amplitude for the resonant scattering channel [see (2)] the Feynman graphs depicted in Fig. 6 have to be taken into account. Figures 6(a) and 6(c) present the direct and exchange graphs, respectively, of the one-photon exchange. The double line describes an electron in the Furry picture with the potential $V^{\mathrm{F}}=-\alpha Z / r$, the double striped line describes continuum electrons in the Furry picture with the potential $V_{\text {cont }}^{\mathrm{F}}=-\alpha(Z-1) / r$. Figure 6(b) represents the interaction of the incident electron with the potential $\Delta V_{\text {cont }}=V^{\mathrm{F}}-V_{\text {cont }}^{\mathrm{F}}=$ $-\alpha / r$ (i.e., with the potential of the remaining charge of the nucleus).

The contribution of Fig. 6(a) [or Fig. 6(c)] is given by [14]

$$
\begin{aligned}
(I)_{u_{1} u_{2} d_{1} d_{2}}= & \alpha \int d \boldsymbol{r}_{1} d \boldsymbol{r}_{2} \bar{\psi}_{u_{1}}\left(\boldsymbol{r}_{1}\right) \bar{\psi}_{u_{2}}\left(\boldsymbol{r}_{2}\right) \gamma_{1}^{\mu_{1}} \gamma_{2}^{\mu_{2}} I_{\mu_{1} \mu_{2}}\left(|\Omega|, r_{12}\right) \\
& \times \psi_{d_{1}}\left(\boldsymbol{r}_{1}\right) \psi_{d_{2}}\left(\boldsymbol{r}_{2}\right)
\end{aligned}
$$

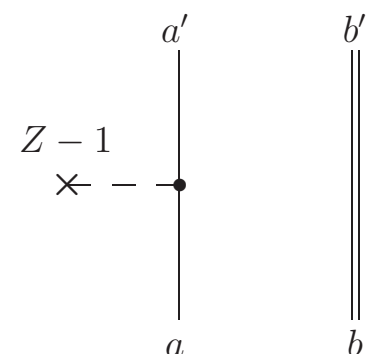

(a)

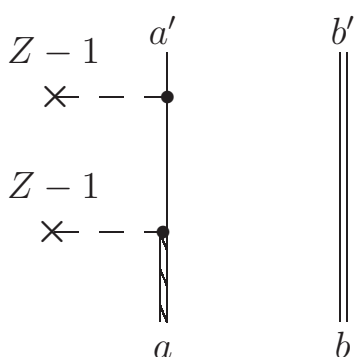

(b)
FIG. 5. Feynman graphs representing the first (a) and second (b) terms in the right side of Eq. (15) for two-electron ion. The single solid line denotes free electron, the double line denotes electron in the field of potential $V^{\mathrm{F}}=-\alpha Z / r$. The dashed line with the cross designates interaction with external field $V_{\text {cont }}=-\alpha(Z-1) / r$. The double striped line describes electron in the Furry picture with potential $V_{\mathrm{cont}}^{\mathrm{F}}=-\alpha(Z-1) / r$.

where $r_{12}=\left|\boldsymbol{r}_{1}-\boldsymbol{r}_{2}\right|$ and

$$
\begin{gathered}
I_{\mu_{1} \mu_{2}}^{\mathrm{c}}=\frac{\delta_{\mu_{1} 0} \delta_{\mu_{2} 0}}{r_{12}}, \\
I_{\mu_{1} \mu_{2}}^{\mathrm{t}}=-\left(\frac{\delta_{\mu_{1} \mu_{2}}}{r_{12}} e^{i \Omega r_{12}}+\frac{\partial}{\partial x_{1}^{\mu_{1}}} \frac{\partial}{\partial x_{2}^{\mu_{2}}} \frac{1-e^{i \Omega r_{12}}}{r_{12} \Omega^{2}}\right) \\
\times\left(1-\delta_{\mu_{1} 0}\right)\left(1-\delta_{\mu_{2} 0}\right) .
\end{gathered}
$$

While the exchange contribution [Fig. 6(c)] does not contain any divergence, the direct contribution [Fig. 6(a)] does. However, the contribution to the amplitude given by Fig. 6(b) is also divergent and it turns out that the divergences in Figs. 6(a) and 6(b) cancel each other.

Let us consider this point more in detail. First of all we note that the problem with Fig. 6(a) arises only due to the Coulomb part of the transition matrix element. We now assume that $d_{1}=u_{1}=e_{p, \mu}$ [the incident and scattered electron having the same energy $(\varepsilon)]$ and $d_{2}=u_{2}=1 s$ (the $1 s$ electron) and consider only the above-mentioned part $I^{\mathrm{c}}$ of the transition matrix element

$$
\left(I^{\mathrm{c}}\right)_{u_{1} u_{2} d_{1} d_{2}}=\alpha \int d \boldsymbol{r}_{1} d \boldsymbol{r}_{2} \psi_{u_{1}}^{+}\left(\boldsymbol{r}_{1}\right) \psi_{u_{2}}^{+}\left(\boldsymbol{r}_{2}\right) \frac{1}{r_{12}} \psi_{d_{1}}\left(\boldsymbol{r}_{1}\right) \psi_{d_{2}}\left(\boldsymbol{r}_{2}\right) .
$$

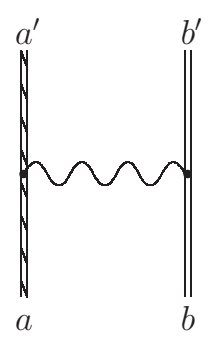

(a)

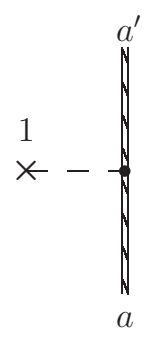

(b)

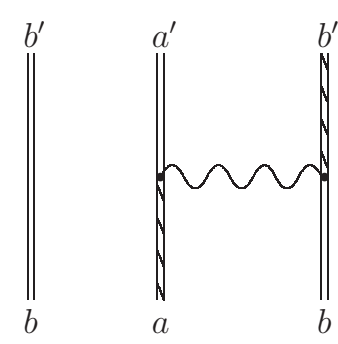

(c)
FIG. 6. The direct (a) and exchange (c) Feynman graphs representing the one-photon exchange. The double line describes electron in the Furry picture with potential $V^{\mathrm{F}}=-\alpha Z / r$, the double striped line describes electron in the Furry picture with potential $V_{\text {cont }}^{\mathrm{F}}=$ $-\alpha(Z-1) / r$. The graph (b) represents interaction of the incident electron with the potential $\Delta V_{\text {cont }}=-\alpha / r$. 
Using the expansion [13]

$$
\frac{1}{r_{12}}=\sum_{K=0}^{\infty} \frac{r_{<}^{K}}{r_{>}^{K+1}} P_{K}(\cos \theta)
$$

where $r_{<}=\min \left(r_{1}, r_{2}\right), r_{>}=\max \left(r_{1}, r_{2}\right)$ and retaining only the term with $K=0$ (the terms with $K>0$ have no divergences) we can write

$$
\left(I_{0}^{\mathrm{c}}\right)_{u_{1} u_{2} d_{1} d_{2}}=\alpha \int d \boldsymbol{r}_{1} d \boldsymbol{r}_{2} \psi_{u_{1}}^{+}\left(\boldsymbol{r}_{1}\right) \psi_{u_{2}}^{+}\left(\boldsymbol{r}_{2}\right) \frac{1}{r_{>}} \psi_{d_{1}}\left(\boldsymbol{r}_{1}\right) \psi_{d_{2}}\left(\boldsymbol{r}_{2}\right)
$$

The wave function of an electron with a given momentum and polarization can be decomposed into the complete set of partial waves with a certain energy and angular momentum [see Eq. (3)]. The divergence appears only for the case of identical partial waves for the incident and scattered electrons, which we shall now consider. The wave function of $1 s$ electron decreases exponentially, accordingly, the integration over $\boldsymbol{r}_{2}$ is convergent. Asymptotically (when $r \rightarrow \infty$ ), the upper and lower components of the incident electron wave function are given by

$$
\begin{aligned}
& g_{\varepsilon j l m}(r) \sim C_{g} \cos \left(p r+\xi \log 2 p r+\delta_{j l}\right), \\
& f_{\varepsilon j l m}(r) \sim C_{f} \sin \left(p r+\xi \log 2 p r+\delta_{j l}\right),
\end{aligned}
$$

where $\xi=\alpha Z \varepsilon / p$ is the Sommerfeld parameter, $\delta_{j l}$ are the phase shifts, $C_{g}$ and $C_{f}$ are constants [12]. Accordingly, the integration over $\boldsymbol{r}_{1}$ contains a divergent part at the upper limit $\left(r_{1} \rightarrow \infty\right)$ :

$$
\int d r_{1} \frac{1}{r_{1}}\left[\left|C_{g}\right|^{2} \cos ^{2}\left(p r_{1}+\xi \log 2 p r_{1}+\delta_{j l}\right)+\left|C_{f}\right|^{2} \sin ^{2}\left(p r_{1}+\xi \log 2 p r_{1}+\delta_{j l}\right)\right] .
$$

After a regularization of the integral over $r_{1}$, the divergent part of Eq. (55) can be singled out and, as will be shown, it will be exactly canceled by the divergence contained in the contribution to the amplitude due to the interaction of the continuum electron with the potential $\Delta V_{\text {cont }}$.

It is convenient to split the integral $I_{0}^{\mathrm{c}}$ into two parts:

$$
\begin{aligned}
\left(I_{0}^{\mathrm{c}}\right)_{u_{1} u_{2} d_{1} d_{2}} & =\alpha \int d \boldsymbol{r}_{1} d \boldsymbol{r}_{2}\left(\frac{1}{r_{>}}-\frac{1}{r_{1}}\right)\left\langle\psi_{u_{1}}^{+}\left(\boldsymbol{r}_{1}\right) \psi_{d_{1}}\left(\boldsymbol{r}_{1}\right)\right\rangle\left\langle\psi_{u_{2}}^{+}\left(\boldsymbol{r}_{2}\right) \psi_{d_{2}}\left(\boldsymbol{r}_{2}\right)\right\rangle+\alpha \int d \boldsymbol{r}_{1} d \boldsymbol{r}_{2}\left(\frac{1}{r_{1}}\right)\left\langle\psi_{u_{1}}^{+}\left(\boldsymbol{r}_{1}\right) \psi_{d_{1}}\left(\boldsymbol{r}_{1}\right)\right\rangle\left\langle\psi_{u_{2}}^{+}\left(\boldsymbol{r}_{2}\right) \psi_{d_{2}}\left(\boldsymbol{r}_{2}\right)\right\rangle \\
& =\alpha \int d \boldsymbol{r}_{2} \int_{\left|\boldsymbol{r}_{1}\right|<\left|\boldsymbol{r}_{2}\right|} d \boldsymbol{r}_{1}\left(\frac{1}{r_{2}}-\frac{1}{r_{1}}\right)\left\langle\psi_{u_{1}}^{+}\left(\boldsymbol{r}_{1}\right) \psi_{d_{1}}\left(\boldsymbol{r}_{1}\right)\right\rangle\left\langle\psi_{u_{2}}^{+}\left(\boldsymbol{r}_{2}\right) \psi_{d_{2}}\left(\boldsymbol{r}_{2}\right)\right\rangle+\alpha \int d \boldsymbol{r}_{1}\left(\frac{1}{r_{1}}\right)\left\langle\psi_{u_{1}}^{+}\left(\boldsymbol{r}_{1}\right) \psi_{d_{1}}\left(\boldsymbol{r}_{1}\right)\right\rangle \delta_{u_{2} d_{2}} .
\end{aligned}
$$

Here, we used the fact that the wave function of the $1 s$ electron is normalized to unity. The integral containing the term $\left(1 / r_{2}-\right.$ $\left.1 / r_{1}\right)$ is obviously convergent. The integral with $1 / r_{1}$ is divergent but is exactly canceled out by the divergent part of the contribution to the amplitude due to the interaction $\Delta V_{\text {cont }}$ of the incident electron with the remaining charge, which reads as

$$
\left(\Delta V_{\text {cont }}\right)_{u_{1} u_{2} d_{1} d_{2}}=\alpha \int d \boldsymbol{r}_{1} \psi_{u_{1}}^{+}\left(\boldsymbol{r}_{1}\right)\left(\frac{-1}{r_{1}}\right) \psi_{d_{1}}\left(\boldsymbol{r}_{1}\right) \delta_{u_{2} d_{2}} .
$$

The consideration given in the previous paragraphs is based on the decomposition $Z=(Z-1)+1$. Its first term corresponds to the effective charge of the nucleus (as seen asymptotically by the incident and scattered electron) screened by the bound electron which essentially is regarded as a pointlike charge $(-1)$ placed at the origin. Using the Furry picture, the Coulomb interaction between the continuum electron and such a pointlike electron is taken into account to all orders. At the same time, the difference between this interaction and the Coulomb interaction between the continuum electron and the bound electron in the $1 s$ state is considered in the first order. We note that this difference is of short range corresponding to the interaction with an electrically neutral system, which is considered using the perturbation theory. Indeed, the sum of $I_{0}^{\mathrm{c}}$ and $\Delta V_{\text {cont }}$

$$
\left(I_{0}^{\mathrm{c}}+\Delta V_{\text {cont }}\right)_{u_{1} u_{2} d_{1} d_{2}}=\alpha \int d \boldsymbol{r}_{2} \int_{\left|\boldsymbol{r}_{1}\right|<\left|\boldsymbol{r}_{2}\right|} d \boldsymbol{r}_{1}\left(\frac{1}{r_{>}}-\frac{1}{r_{1}}\right)\left\langle\psi_{u_{1}}^{+}\left(\boldsymbol{r}_{1}\right) \psi_{d_{1}}\left(\boldsymbol{r}_{1}\right)\right\rangle\left\langle\psi_{u_{2}}^{+}\left(\boldsymbol{r}_{2}\right) \psi_{d_{2}}\left(\boldsymbol{r}_{2}\right)\right\rangle
$$

is finite and represents a correction due to interaction with a short-range potential. Within our approach, Fig. 6(a) must be always considered together with Fig. 6(b). For light ions the accuracy of our approach may be improved if we replace the interaction with a pointlike charge by the interaction with the charge density given by the $1 s$ electron wave function and adjust the Furry picture accordingly. The influence of this replacement on the results is discussed in the next section (Figs. 11 and 12).

The Feynman graph depicted in Fig. 6(c) represents the exchange graph of the one-photon exchange, it is finite and does not require a special treatment. Up to now, we considered the direct (nonresonant) elastic scattering. Let us now briefly discuss the description of the resonant channel of the scattering process which becomes relevant when the sum of the energies of the incident and the bound electrons is close to the energy of a doubly excited (autoionizing) state. In such a case, the contribution of two- and more-photon exchange between electrons in low-lying states becomes of importance. Moreover, the doubly excited states are normally quasidegenerate, hence, a perturbation theory for quasidegenerate states has to be used. For this purpose, the LPA [14] is employed.

For application of the quasidegenerate perturbation theory within the LPA we introduce the set of two-electron 
configurations $(g)$ in the $j-j$ coupling scheme which includes all two-electron configurations composed by a certain set of electrons (for example, $1 s, 2 s, 2 p, 3 s, 3 p, 3 d$ electrons)

$$
\begin{aligned}
& \Psi_{J M j_{1} j_{2} l_{1} l_{2} n_{1} n_{2}}^{(0)}\left(\boldsymbol{r}_{1}, \boldsymbol{r}_{2}\right) \\
& \quad=N^{\prime} \sum_{m_{1} m_{2}} C_{J M}^{j_{1} j_{2}}\left(m_{1}, m_{2}\right) \operatorname{det}\left\{\psi_{n_{1} j_{1} l_{1} m_{1}}\left(\boldsymbol{r}_{1}\right), \psi_{n_{2} j_{2} l_{2} m_{2}}\left(\boldsymbol{r}_{2}\right)\right\},
\end{aligned}
$$

where $j$ and $m$ are the total angular momentum and its projection, $l$ is the orbital angular momentum defining the parity, $n$ denotes the principal quantum number or energy (for the continuum electrons), $J$ and $M$ are the total angular momentum of the two-electron configuration and its projection, $N^{\prime}$ is the normalizing constant.

In the LPA a matrix $V$ is introduced, which is determined by the one- and two-photon exchange, electron self-energy and vacuum polarization matrix elements and other QED corrections [14] and which can be derived order by order within the QED perturbation theory. The matrix $V=V^{(0)}+\Delta V$ is considered as a block matrix

$$
V=\left[\begin{array}{ll}
V_{11} & V_{12} \\
V_{21} & V_{22}
\end{array}\right]=\left[\begin{array}{cc}
V_{11}^{(0)}+\Delta V_{11} & \Delta V_{12} \\
\Delta V_{21} & V_{22}^{(0)}+\Delta V_{22}
\end{array}\right] \text {. }
$$

The matrix $V_{11}$ is defined on the set $g$, which contains configurations mixing with the reference state $n_{g} \in g$. The matrix $V^{(0)}$ is a diagonal matrix composed of the sum of the one-electron Dirac energies. The matrix $\Delta V_{11}$ is not a diagonal matrix, but it contains a small parameter of the QED perturbation theory. The matrix $V_{11}$ is a finite-dimensional matrix and can be diagonalized numerically:

$$
V_{11}^{\text {diag }}=B^{t} V_{11} B, \quad B^{t} B=I .
$$

The matrix $B$ defines a transformation to the basis set in which the matrix $V_{11}$ is a diagonal matrix. Then, the standard perturbation theory can be applied for the diagonalization of the infinite-dimensional matrix $V$. The eigenvectors of $V$ read as [14]

$$
\Phi_{n_{g}}=\sum_{k_{g} \in g} B_{k_{g} n_{g}} \Psi_{k_{g}}^{(0)}+\sum_{k \notin g, l_{g} \in g}\left[\Delta V_{21}\right]_{k l_{g}} \frac{B_{l_{g} n_{g}}}{E_{n_{g}}^{(0)}-E_{k}^{(0)}} \Psi_{k}^{(0)}+\cdots,
$$

where $n_{g} \equiv\left(J M j_{1} j_{2} l_{1} l_{2} n_{1} n_{2}\right)$ is a complete set of quantum numbers describing the reference state, indices $k, l_{g}$ denote the two-electron configurations: the index $l_{g}$ runs over all configurations of the set $g$; the index $k$ runs over all configurations not included in the set $g$ (this implies the integration over the positive- and negative-energy continuum). Here, $E_{k}^{(0)}$ is the energy of the two-electron configuration $\Psi_{k}^{(0)}$ given by the sum of the one-electron Dirac energies.

The amplitude of the scattering process is given as a matrix element of the operator $(\Delta \hat{V})$

$$
U_{i f}^{\text {Auger }}=\left\langle\Psi_{\text {fin }}^{(0)}|\Delta \hat{V}| \Phi_{\text {ini }}\right\rangle .
$$

The bra vector corresponds to the wave function describing noninteracting electrons, Eq. (62), the ket vector is given by the eigenvector, Eq. (65). The operator $\Delta \hat{V}$ is derived within the QED perturbation theory order by order [14,24]. In the first and second orders of the perturbation theory it is represented by Feynman graphs depicted in Figs. 3 and 4, respectively.

The total amplitude of the process (1), including its resonant part (1), is given by

$$
U_{i f}=U_{i f}^{\text {Coul }}+U_{i f}^{\text {Auger }},
$$

where the Coulomb and Auger contributions are given by Eqs. (19) and (66), respectively. The numerical calculation of the Coulomb amplitude $U_{\text {if }}^{\text {Coul }}$ is discussed in Appendix B.

The transition probability is expressed via the amplitude according to [12]

$$
d w_{i f}=2 \pi\left|U_{i f}\right|^{2} \delta\left(E_{i}-E_{f}\right) \frac{d^{3} \boldsymbol{p}_{f}}{(2 \pi)^{3}},
$$

where $E_{i}, E_{f}$ are the energies of the initial and final states of the system and $\boldsymbol{p}_{f}$ is the momentum of the scattered electron.

The cross section is defined as

$$
d \sigma_{i f}=\frac{d w_{i f}}{j},
$$

where $j=p_{i} / \varepsilon_{i}$ is the flux of the incident electrons having an energy $\varepsilon_{i}$ and a momentum $p_{i}$. Accordingly, the double and single differential cross sections for elastic electron scattering read as

$$
\begin{gathered}
\frac{d \sigma_{i f}}{d \varepsilon_{f} d \Omega_{f}}\left(\varepsilon_{f}, \theta_{f}\right)=2 \pi\left|U_{i f}\right|^{2} \delta\left(\varepsilon_{f}-\varepsilon_{i}\right) \frac{\varepsilon_{i}}{p_{i}} \frac{p_{f} \varepsilon_{f}}{(2 \pi)^{3}}, \\
\frac{d \sigma_{i f}}{d \Omega_{f}}\left(\varepsilon_{f}=\varepsilon_{i}, \theta_{f}\right)=2 \pi\left|U_{i f}\right|^{2} \frac{\varepsilon_{i}}{p_{i}} \frac{p_{f} \varepsilon_{f}}{(2 \pi)^{3}},
\end{gathered}
$$

where $\varepsilon_{f}$ and $\Omega_{f}$ are the energy and solid angle (with polar angle $\theta_{f}$ ) of the scattered electron, respectively.

\section{RESULTS AND DISCUSSION}

In this section we discuss results of applications of our theory to electron scattering on hydrogen-like ions ranging from boron to uranium. The calculated cross sections will be given in the rest frame of the ion and for those impact energies where only $L L$ autoionizing states participate in the scattering process. Since the Coulomb scattering is especially strong in forward angles, we restrict ourselves to the consideration of electron scattering to backward angles for which the Coulomb contribution is minimal.

In experiments on electron-ion scattering free electrons are often replaced by electrons bound in light atomic (molecular) targets which serve as a source of (quasi)free electrons. Therefore, in what follows we consider collisions of hydrogen-like ions not only with free electrons, but also with molecular hydrogen.

In the bottom panels of Figs. 7-10 the single differential cross section for scattering of free electrons is presented as a function of the scattered electron kinetic energy for $\mathrm{Ca}^{19+}(1 s)$, $\mathrm{Fe}^{25+}(1 s), \mathrm{Kr}^{35+}(1 s)$, and $\mathrm{Xe}^{53+}(1 s)$. In these figures we observe a smooth background, caused by Coulomb scattering, which is superimposed by maxima and minima arising due to the resonant scattering as well as interference between the resonant and the Coulomb parts of the scattering process. The figures show that the differential cross section strongly 


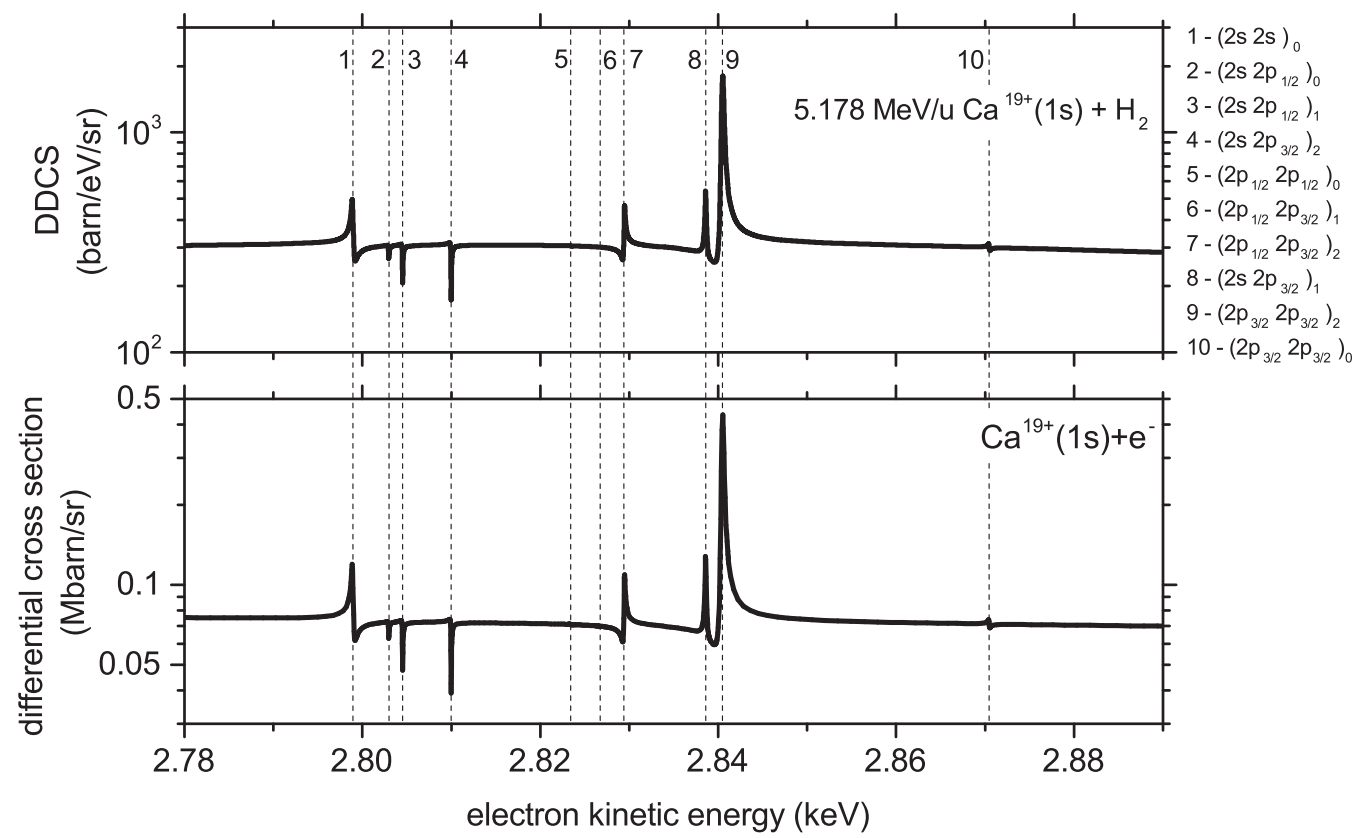

FIG. 7. Double-differential cross section for the elastic electron scattering in collisions of $5.178 \mathrm{MeV} / \mathrm{u} \mathrm{Ca}^{19+}(1 s)$ with $\mathrm{H}_{2}$ target (top) and single differential cross section for the elastic electron scattering in collisions of $\mathrm{Ca}^{19+}(1 s)$ with free electrons (bottom).

depends on the charge of the ionic nucleus $(Z)$ both qualitatively and quantitatively.

The total scattering amplitude can be written as a sum of the two parts: the nonresonant (Coulomb) part and the resonant part. Accordingly, the cross section can be split into three terms corresponding to the Coulomb scattering, the resonant scattering, and their interference. When the charge $Z$ of the ionic nucleus varies, the amplitude for Coulomb scattering in the vicinity of resonances effectively scales as
$1 / Z$. Further, the Schrödinger equation predicts that, provided the total width of an autoionizing state is determined mainly by the electron-electron interaction, the amplitude for resonant scattering scales as $1 / Z$ as well. Therefore, for not too heavy ions one could expect that all the contributions to the cross section, the Coulomb and the resonant parts and the interference term, scale with $Z$ roughly as $1 / Z^{2}$. Indeed, our calculations for ions ranging between $Z \sim 5$ and $Z \sim 20$ are in qualitative agreement with this scaling (for

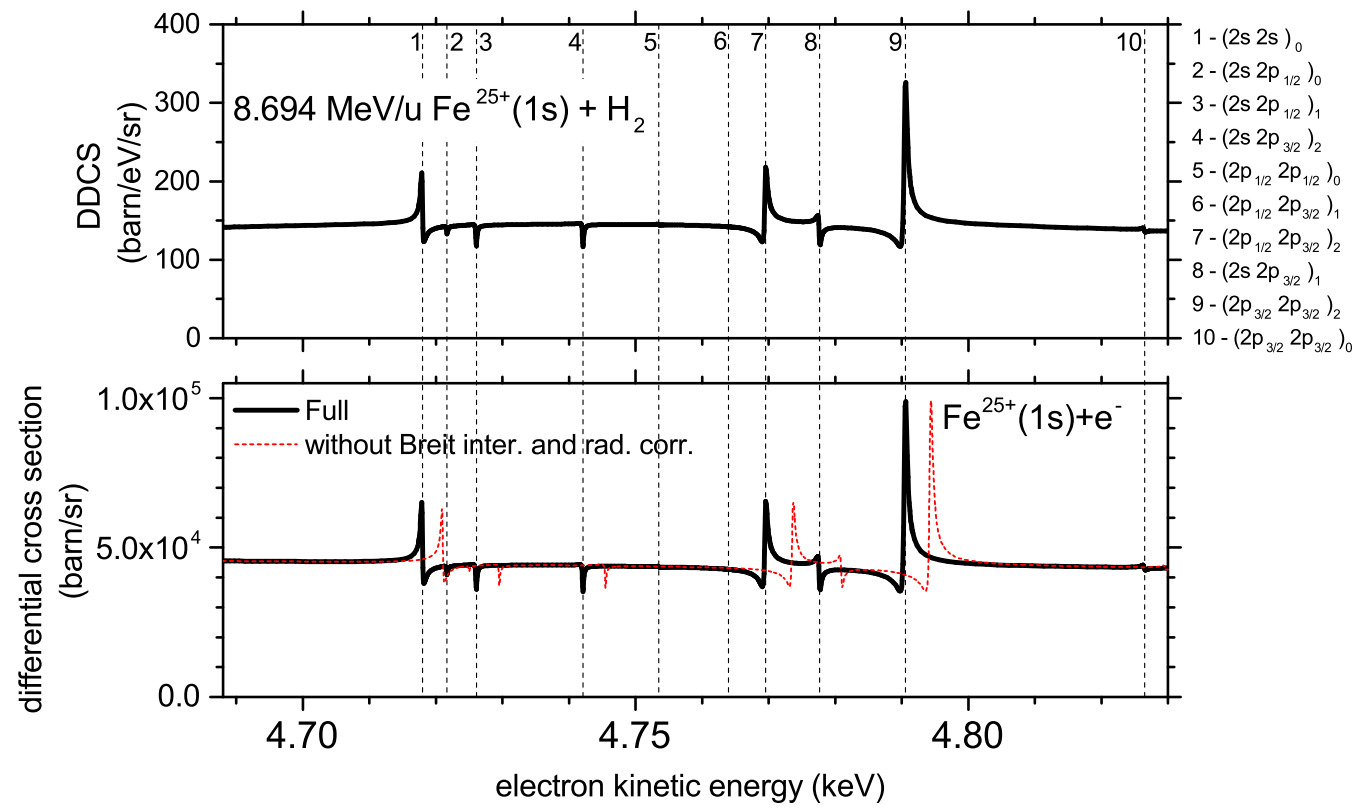

FIG. 8. The same as in Fig. 7 but for $8.694 \mathrm{MeV} / \mathrm{u} \mathrm{Fe}^{25+}(1 \mathrm{~s})$. The solid black lines represent the results of the exact QED calculation, the dashed red line corresponds to the calculation with disregard of the Breit part of the interelectron interaction and the real part of the radiative corrections. 


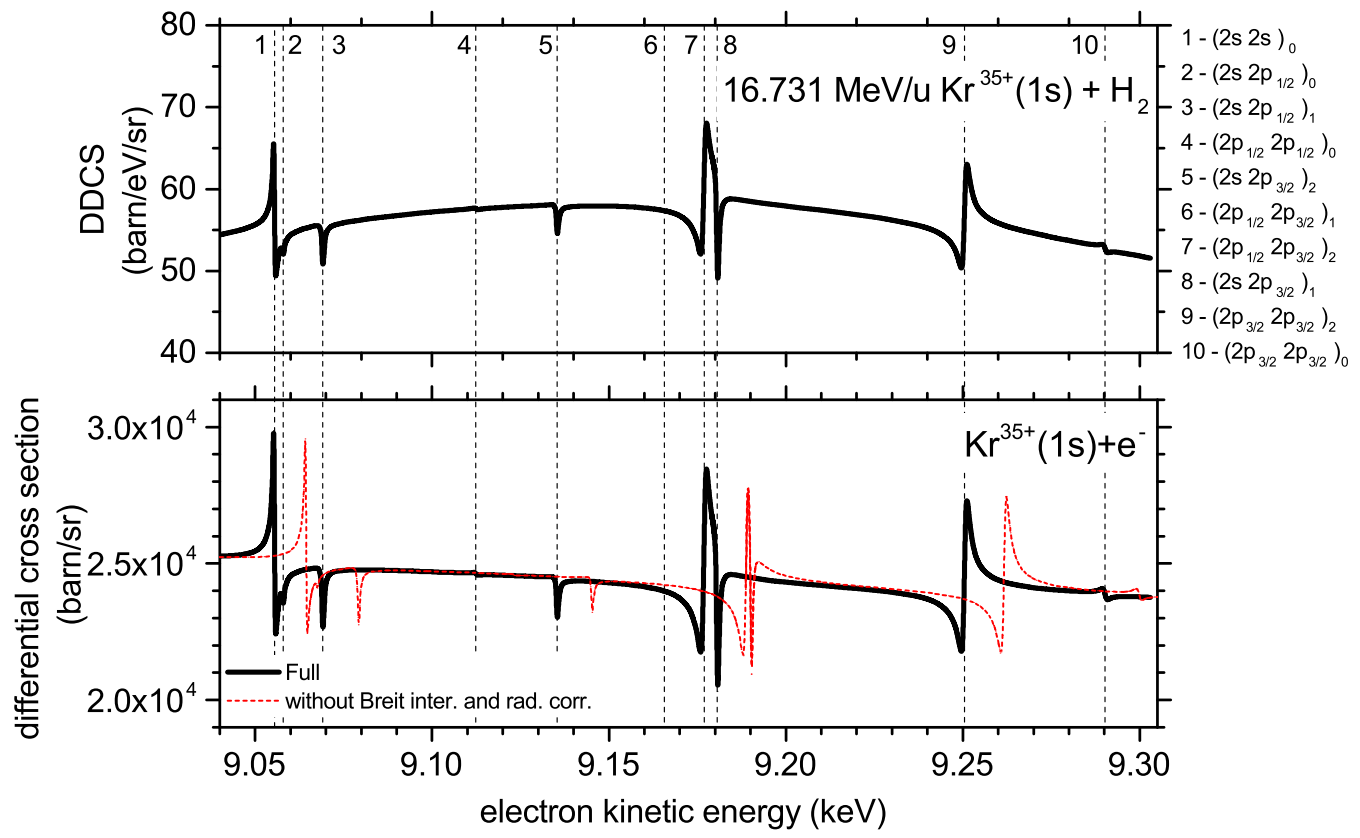

FIG. 9. The same as in Fig. 8 but for $16.731 \mathrm{MeV} / \mathrm{u} \mathrm{Kr}^{35+}(1 s)$.

illustration see Figs. 7 and 11). For heavier ions the amplitude for the resonant scattering begins to decrease with $Z$ faster than $1 / Z$. This is mainly caused by a rapid growth of the radiative contribution to the total widths which in heavy ions outperforms the Auger decay. Accordingly, with increasing $Z$ the contribution of the resonant scattering decreases faster than the contribution from the interference term. Whereas for relatively small $Z$ all the three parts of the cross section are equally important, for higher $Z$ the purely resonant part becomes of minor importance and the resonance structure in the cross section is determined solely by the interference term.

We also note that the order of resonances also depends on $Z$, in particular, it leads to different orders of maxima and minima for different $Z$. The resonant energies of the incident electron as well as the energies and widths of the corresponding autoionizing states for various ions are presented in Tables I-IV.

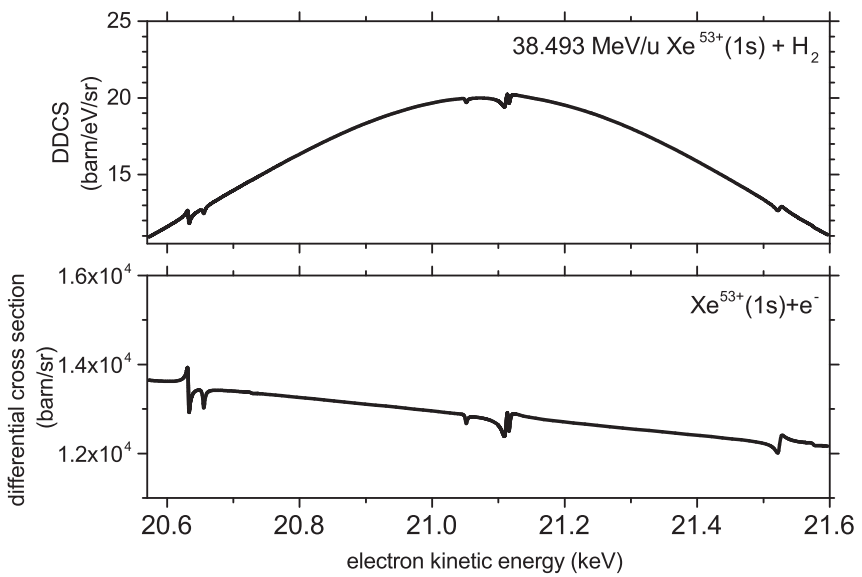

FIG. 10. The same as in Fig. 7 but for $38.493 \mathrm{MeV} / \mathrm{u} \mathrm{Xe}^{53+}(1 s)$.
In order to investigate the influence of the Breit interaction and the radiative corrections (self-energy and vacuum polarization), we performed calculations where these corrections were omitted. The red dotted lines in the bottom panels of Figs. 8 and 9 correspond to calculations in which the Breit interaction and the radiative corrections were neglected. The energy shift of the resonances is clearly visible and roughly equals to $\sim 4$ and $\sim 10 \mathrm{eV}$ for iron and krypton, respectively. A slight decrease in the differential cross section due to the contribution of the Breit interaction is noticeable but turns out to be rather small even for krypton.

In the top panels of Figs. 7-10 we present results for collisions of highly charged ions with $\mathrm{H}_{2}$. In order to evaluate

TABLE I. The parameters characterizing the resonant structure of the cross section for various autoionizing $(L L)$ states of heliumlike calcium are presented. The second and third columns present the energies $\left(E^{\prime}=E_{(L L)}-2 m c^{2}\right)$ and widths $(\Gamma)$ of the autoionizing states. The fourth column presents the resonant energies of the scattered electron $\left(\varepsilon_{\mathrm{res}}=E_{(L L)}-\varepsilon_{1 s}\right)$. The data are given in the rest frame of the ion.

\begin{tabular}{lccc}
\hline \hline $\begin{array}{l}\text { Autoionizing } \\
\text { state }\end{array}$ & $E^{\prime}(\mathrm{keV})$ & $\Gamma(\mathrm{eV})$ & $\varepsilon_{\text {res }}(\mathrm{keV})$ \\
\hline$(2 s)_{0}^{2}$ & -2.6710 & 0.25 & 2.7989 \\
$\left(2 s 2 p_{1 / 2}\right)_{0}$ & -2.6670 & 0.08 & 2.8030 \\
$\left(2 s 2 p_{1 / 2}\right)_{1}$ & -2.6654 & 0.08 & 2.8045 \\
$\left(2 s 2 p_{3 / 2}\right)_{2}$ & -2.6600 & 0.07 & 2.8100 \\
$\left(2 p_{1 / 2}\right)_{0}^{2}$ & -2.6465 & 0.13 & 2.8234 \\
$\left(2 p_{1 / 2} 2 p_{3 / 2}\right)_{1}$ & -2.6432 & 0.13 & 2.8267 \\
$\left(2 p_{1 / 2} 2 p_{3 / 2}\right)_{2}$ & -2.6405 & 0.16 & 2.8294 \\
$\left(2 s 2 p_{3 / 2}\right)_{1}$ & -2.6313 & 0.19 & 2.8386 \\
$\left(2 p_{3 / 2}\right)_{2}^{2}$ & -2.6295 & 0.33 & 2.8405 \\
$\left(2 p_{3 / 2}\right)_{0}^{2}$ & -2.5995 & 0.12 & 2.8705 \\
\hline \hline
\end{tabular}


TABLE II. Same as in Table I but for helium-like iron.

\begin{tabular}{lccc}
\hline \hline $\begin{array}{l}\text { Autoionizing } \\
\text { state }\end{array}$ & $E^{\prime}(\mathrm{keV})$ & $\Gamma(\mathrm{eV})$ & $\varepsilon_{\text {res }}(\mathrm{keV})$ \\
\hline$(2 s)_{0}^{2}$ & -4.5597 & 0.31 & 4.7179 \\
$\left(2 s 2 p_{1 / 2}\right)_{0}$ & -4.5560 & 0.21 & 4.7216 \\
$\left(2 s 2 p_{1 / 2}\right)_{1}$ & -4.5516 & 0.20 & 4.7260 \\
$\left(2 s 2 p_{3 / 2}\right)_{2}$ & -4.5356 & 0.19 & 4.7421 \\
$\left(2 p_{1 / 2}\right)_{0}^{2}$ & -4.5242 & 0.35 & 4.7534 \\
$\left(2 p_{1 / 2} 2 p_{3 / 2}\right)_{1}$ & -4.5138 & 0.37 & 4.7639 \\
$\left(2 p_{1 / 2} 2 p_{3 / 2}\right)_{2}$ & -4.5082 & 0.45 & 4.7695 \\
$\left(2 s 2 p_{3 / 2}\right)_{1}$ & -4.5001 & 0.31 & 4.7776 \\
$\left(2 p_{3 / 2}\right)_{2}^{2}$ & -4.4872 & 0.53 & 4.7905 \\
$\left(2 p_{3 / 2}\right)_{0}^{2}$ & -4.4513 & 0.34 & 4.8264 \\
\hline \hline
\end{tabular}

the doubly differential scattering cross section for collisions with molecular hydrogen, we use the impulse approximation where the electrons, which are initially bound in hydrogen, are considered as quasifree. Using this approximation one can show that the cross section in collisions with hydrogen can be expressed via the cross section in collisions with free electrons according to

$$
\left(\frac{d^{2} \sigma\left(\varepsilon_{f}, \theta_{f}\right)}{d \varepsilon_{f} d \Omega_{f}}\right)_{H_{2}}=2\left(\frac{d \sigma\left(\varepsilon_{f}, \theta_{f}\right)}{d \Omega_{f}}\right)_{\text {free }} \frac{\varepsilon_{f}}{\gamma p_{f}} J\left(p_{z}^{\prime}\right) .
$$

Here, $\gamma=1 / \sqrt{1-V_{\text {col }}^{2}}$ and $p_{z}^{\prime}=\gamma\left(p_{f}-V_{\text {col }} \varepsilon_{f}\right)$, where $V_{\text {col }}$ is the collision velocity. Further,

$$
\begin{aligned}
J\left(p_{z}^{\prime}\right) & =\int d^{2} \mathbf{p}_{\perp}^{\prime}\left|\psi_{1 s}\left(\mathbf{p}_{\perp}^{\prime}, p_{z}^{\prime}\right)\right|^{2} \\
& =\frac{8}{3 \pi} \frac{\alpha^{5}}{\left(\alpha^{2}+p_{z}^{\prime 2}\right)^{3}}
\end{aligned}
$$

is the Compton profile for the $1 s$ electron of hydrogen atom with the corresponding wave function $\psi_{1 s}\left(\mathbf{p}^{\prime}\right), \mathbf{p}^{\prime}=\left(\mathbf{p}_{\perp}^{\prime}, p_{z}^{\prime}\right)$ is the momentum of the incident electron in the rest frame of hydrogen, $\alpha$ is the hyperfine structure constant (which corresponds to the characteristic orbiting momentum of the $1 s$ electron in hydrogen expressed in relativistic units), and $\left(\frac{d \sigma\left(\varepsilon_{f}, \theta_{f}\right)}{d \Omega_{f}}\right)_{\text {free }}$ is the cross section for collision with a free electron given by Eq. (71).

TABLE III. Same as in Table I but for helium-like krypton.

\begin{tabular}{lccc}
\hline \hline $\begin{array}{l}\text { Autoionizing } \\
\text { state }\end{array}$ & $E^{\prime}(\mathrm{keV})$ & $\Gamma(\mathrm{eV})$ & $\varepsilon_{\text {res }}(\mathrm{keV})$ \\
\hline$(2 s)_{0}^{2}$ & -8.8807 & 0.551 & 9.0555 \\
$\left(2 s 2 p_{1 / 2}\right)_{0}$ & -8.8782 & 0.724 & 9.0579 \\
$\left(2 s 2 p_{1 / 2}\right)_{1}$ & -8.8670 & 0.725 & 9.0691 \\
$\left(2 p_{1 / 2}\right)_{0}^{2}$ & -8.8237 & 1.133 & 9.1124 \\
$\left(2 s 2 p_{3 / 2}\right)_{2}$ & -8.8008 & 0.687 & 9.1354 \\
$\left(2 p_{1 / 2} 2 p_{3 / 2}\right)_{1}$ & -8.7705 & 1.366 & 9.1657 \\
$\left(2 p_{1 / 2} 2 p_{3 / 2}\right)_{2}$ & -8.7593 & 1.494 & 9.1769 \\
$\left(2 s 2 p_{3 / 2}\right)_{1}$ & -8.7555 & 0.792 & 9.1806 \\
$\left(2 p_{3 / 2}\right)_{2}^{2}$ & -8.6856 & 1.453 & 9.2505 \\
$\left(2 p_{3 / 2}\right)_{0}^{2}$ & -8.6459 & 1.316 & 9.2902 \\
\hline \hline
\end{tabular}

TABLE IV. Same as in Table I but for helium-like xenon.

\begin{tabular}{lccc}
\hline \hline $\begin{array}{l}\text { Autoionizing } \\
\text { state }\end{array}$ & $E^{\prime}(\mathrm{keV})$ & $\Gamma(\mathrm{eV})$ & $\varepsilon_{\text {res }}(\mathrm{keV})$ \\
\hline$\left(2 s 2 p_{1 / 2}\right)_{0}$ & -20.669 & 3.638 & 20.632 \\
$(2 s)_{0}^{2}$ & -20.669 & 2.265 & 20.632 \\
$\left(2 s 2 p_{1 / 2}\right)_{1}$ & -20.646 & 3.617 & 20.655 \\
$\left(2 p_{1 / 2}\right)_{0}^{2}$ & -20.574 & 5.166 & 20.726 \\
$\left(2 s 2 p_{3 / 2}\right)_{2}$ & -20.250 & 3.372 & 21.051 \\
$\left(2 p_{1 / 2} 2 p_{3 / 2}\right)_{1}$ & -20.205 & 6.905 & 21.096 \\
$\left(2 p_{1 / 2} 2 p_{3 / 2}\right)_{2}$ & -20.189 & 7.050 & 21.112 \\
$\left(2 s 2 p_{3 / 2}\right)_{1}$ & -20.185 & 3.463 & 21.116 \\
$\left(2 p_{3 / 2}\right)_{2}^{2}$ & -19.777 & 6.784 & 21.524 \\
$\left(2 p_{3 / 2}\right)_{0}^{2}$ & -19.726 & 6.695 & 21.575 \\
\hline \hline
\end{tabular}

Comparing the cross sections for collisions with free and quasifree electrons we can conclude that the resonance structure remains basically the same and the only difference is the bending of the background caused by the convolution with the Compton profile. The bending is more prominent for collisions with heavier ions since the energy interval considered scales with $Z$ as $Z^{2}$.

In our approach, the Coulomb interaction of the incident and scattered electron with the nucleus and partly with the bound $1 s$ electron is taken into account within the Furry picture. In Eq. (60) the interaction of the electron in the continuum with the $1 s$ electron is considered, in the first order of the perturbation theory, as the Coulomb interaction with a pointlike charge located in the origin. This is consistent with the Furry picture for the continuum electrons, in which they are regarded as moving in the field $V_{\text {cont }}^{\mathrm{F}}=-\alpha(Z-1) / r$. Equation (61) represents the difference between the longrange term of the Coulomb interaction of the continuum electron with the $1 s$ electron, given by Eq. (55) [contribution of the term with $K=0$ in Eq. (54)], and the interaction of the continuum electron with the pointlike charge placed in the origin. Hence, Eq. (61) describes the interaction with a shortrange potential in the first order of the perturbation theory. We note that all numerical results presented in Figs. 7-10 were obtained using the Furry picture mentioned above.

In order to investigate the importance of the higher orders of the perturbation theory corresponding to the interaction with this potential, we replaced in Eqs. (60) and (61) the interaction with a pointlike charge by the interaction with a charge density corresponding to the $1 s$ electron wave function

$$
\Delta V_{\text {cont }}=-\alpha \int d \boldsymbol{r}^{\prime} \frac{\psi_{1 s}^{+}\left(\boldsymbol{r}^{\prime}\right) \psi_{1 s}\left(\boldsymbol{r}^{\prime}\right)}{\left|\boldsymbol{r}^{\prime}-\boldsymbol{r}\right|} .
$$

Since the $1 s$ electron wave function is independent of angular variables, with employment of the decomposition (54) the potential (74) can be deduced to

$$
\Delta V_{\text {cont }}(r)=-\alpha \int_{0}^{\infty} d r^{\prime} \frac{\rho\left(r^{\prime}\right)}{r_{>}}
$$

where $\rho\left(r^{\prime}\right)$ is the probability density function of the $1 s$ electron $r_{>}=\max \left(r, r^{\prime}\right)$.

With this replacement the Furry picture should be changed accordingly: now the continuum electron is considered to be 


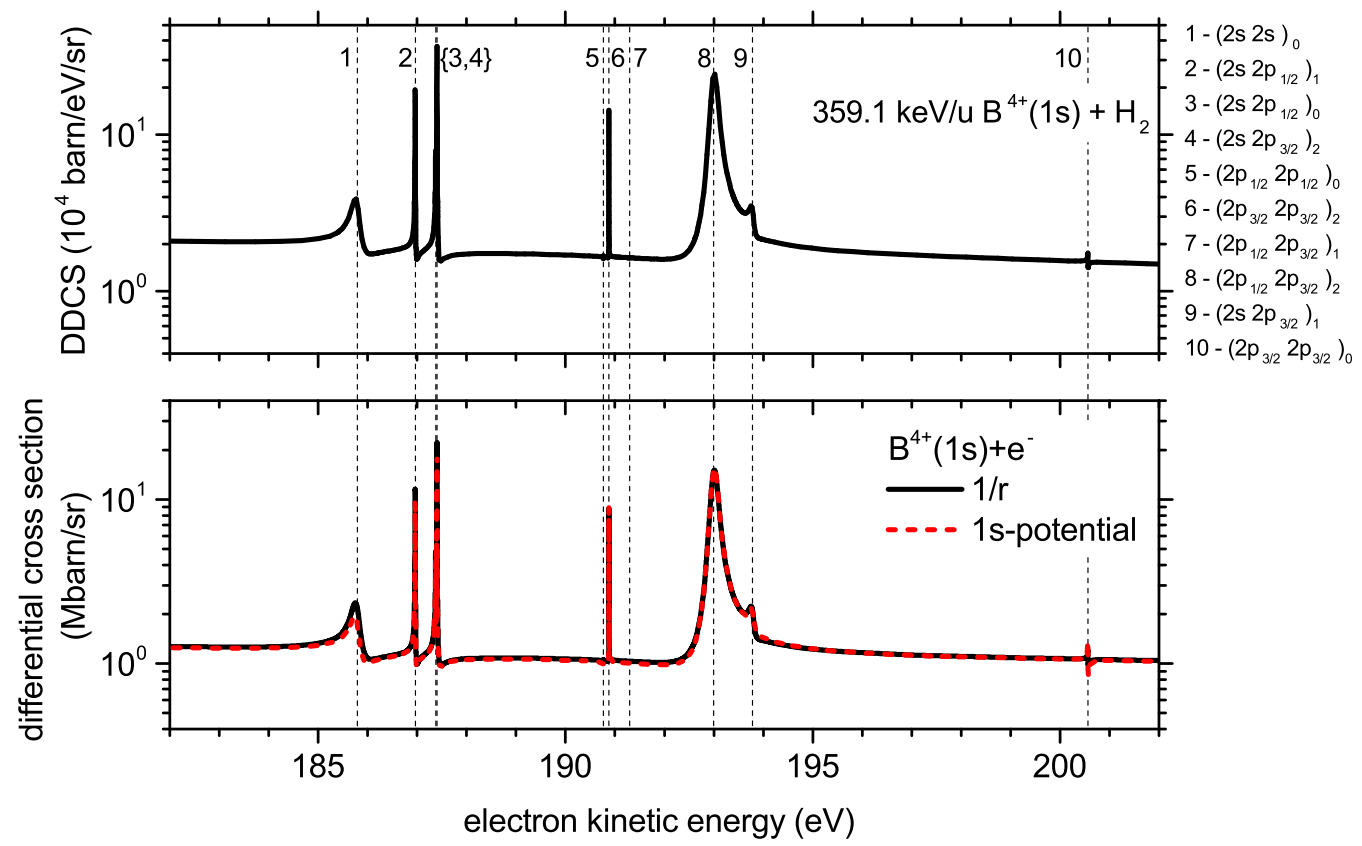

FIG. 11. Same as in Fig. 7 but for $359.1 \mathrm{keV} / \mathrm{u} \mathrm{B}^{4+}(1 s)$. The solid black curves represent results of calculations with potential $\Delta V_{\text {cont }}=$ $-\alpha / r$, the dotted red curve corresponds to the calculation with the potential $\Delta V_{\text {cont }}$ given by Eq. (74).

moving in the potential

$$
V_{\mathrm{cont}}^{\mathrm{F}}=-\frac{\alpha Z}{r}+\alpha \int d \boldsymbol{r}^{\prime} \frac{\psi_{1 s}^{+}\left(\boldsymbol{r}^{\prime}\right) \psi_{1 s}\left(\boldsymbol{r}^{\prime}\right)}{\left|\boldsymbol{r}^{\prime}-\boldsymbol{r}\right|}
$$

Employment of the potential (76) instead of the potential $V_{\text {cont }}^{\mathrm{F}}=-\alpha(Z-1) / r$ yields a correction to the scattering amplitude. This correction is scaled with $Z$ as $1 / Z^{2}$.

In [5] an experimental-theoretical investigation of resonant electron scattering in collisions of hydrogen-like ions $\mathrm{B}^{4+}(1 s)$ of boron with $\mathrm{H}_{2}$ targets was reported. In particular, in [5] results of nonrelativistic calculations within the $R$-matrix approach were presented. In order to make a certain test of our method, we performed calculations for the same scattering system. In Fig. 11 the differential cross section of elastic electron scattering on $\mathrm{B}^{4+}(1 s)$ is presented in the rest frame of the ion. The solid black curve shows our results obtained by using the potential $V_{\text {cont }}^{\mathrm{F}}=-\alpha(Z-1) / r$, the dashed red curve displays the results calculated with the potential (76). By comparing them one can conclude that the higher orders of the perturbation theory corresponding to the interaction (61) give quite a small correction in the case of boron ions and, hence, can be neglected for heavier ions as well.

Comparing our results with those obtained using the nonrelativistic $R$-matrix approach of [5] we see that overall there is a reasonably good agreement between them. Nevertheless, one substantial disagreement should be mentioned: our results show the presence of clear resonances due to the $\left(2 s, 2 p_{1 / 2}\right)_{1}$ and $\left(2 p_{3 / 2}^{2}\right)_{2}$ autoionizing states which are absent in the calculation [5]. It can be explained by the difference between relativistic and nonrelativistic description of the electron states which remains noticeable even for light atomic systems.

For a comparison of our results with the experimental data of [5], the doubly differential cross section for collision with hydrogen (the upper panel of Fig. 11) was convoluted with a
Gaussian function

$$
\begin{aligned}
\left(\frac{d^{2} \sigma\left(\varepsilon_{f}, \theta_{f}\right)}{d \varepsilon_{f} d \Omega_{f}}\right)_{\mathrm{H}_{2}, \text { expt }}= & \frac{1}{\sqrt{2 \pi} \sigma_{\mathrm{r}}} \int d \varepsilon \exp \left(\frac{-\left(\varepsilon-\varepsilon_{f}\right)^{2}}{2 \sigma_{\mathrm{r}}^{2}}\right) \\
& \times\left(\frac{d^{2} \sigma\left(\varepsilon, \theta_{f}\right)}{d \varepsilon d \Omega_{f}}\right)_{\mathrm{H}_{2}},
\end{aligned}
$$

where $\sigma_{\mathrm{r}}=0.56 \mathrm{eV}$ is the experimental resolution. The result of the convolution is presented in Fig. 12. The resonance with the $(2 s)^{2}$ state is clearly seen in our calculations and

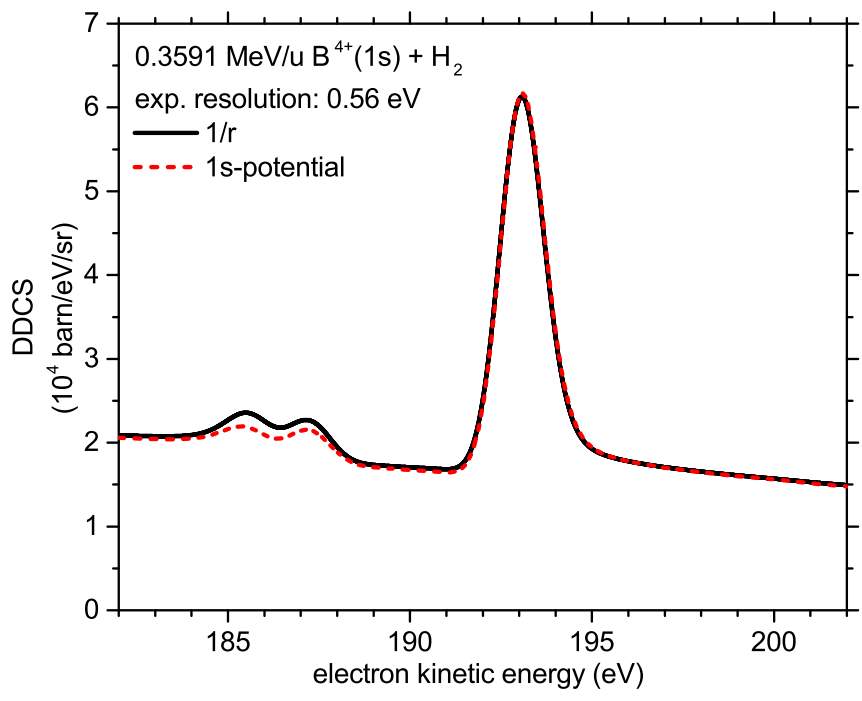

FIG. 12. Double-differential cross section from the top panel of Fig. 11 convoluted with Gaussian [see Eq. (77)]. The solid black curves represent results of calculations with potential $\Delta V_{\text {cont }}=$ $-\alpha / r$, the dotted red curve corresponds to the calculation with the potential $\Delta V_{\text {cont }}$ given by Eq. (74). 
TABLE V. Same as in Table I but for helium-like boron.

\begin{tabular}{lccl}
\hline \hline $\begin{array}{l}\text { Autoionizing } \\
\text { state }\end{array}$ & $E^{\prime}(\mathrm{eV})$ & $\Gamma(\mathrm{eV})$ & $\varepsilon_{\text {res }}(\mathrm{eV})$ \\
\hline$\left(2 s^{2}\right)_{0}$ & -154.5 & 0.195 & 185.8 \\
$\left(2 s^{2}\right)^{1} S$ & & $0.194^{\mathrm{a}}$ & $186.14^{\mathrm{a}}$ \\
$\left(2 s 2 p_{1 / 2}\right)_{1}$ & -153.3 & 0.008 & 187.0 \\
$\left(2 s 2 p_{1 / 2}\right)_{0}$ & -152.9 & 0.008 & 187.4 \\
$\left(2 s 2 p_{3 / 2}\right)_{2}$ & -152.8 & 0.008 & 187.4 \\
$(2 s 2 p)^{3} P$ & & $0.015^{\mathrm{a}}$ & $187.44^{\mathrm{a}}$ \\
$\left(2 p_{1 / 2}^{2}\right)_{0}$ & -149.5 & $<0.001$ & 190.8 \\
$\left(2 p_{3 / 2}^{2}\right)_{2}$ & -149.4 & 0.002 & 190.9 \\
$\left(2 p_{1 / 2} 2 p_{3 / 2}\right)_{1}$ & -148.9 & $<0.001$ & 191.3 \\
$\left(2 p_{1 / 2} 2 p_{3 / 2}\right)_{2}$ & -147.2 & 0.192 & 193.0 \\
$\left(2 p^{2}\right)^{1} D$ & & $0.188^{\mathrm{a}}$ & $193.89^{\mathrm{a}}$ \\
$\left(2 s 2 p_{3 / 2}\right)_{1}$ & -146.5 & 0.103 & 193.8 \\
$(2 s 2 p)^{1} P$ & & $0.088^{\mathrm{a}}$ & $194.20^{\mathrm{a}}$ \\
$\left(2 p_{3 / 2}^{2}\right)_{0}$ & -139.7 & 0.007 & 200.6 \\
$\left(2 p^{2}\right)^{1} S$ & & $0.010^{\mathrm{a}}$ & $200.90^{\mathrm{a}}$ \\
\hline \hline
\end{tabular}

${ }^{\mathrm{a}}$ Reference [5].

in the calculations of [5]; however, it is not observable in the experimental data of [5]. Also, we note that our results obtained using Eq. (76) (the dashed red line in Fig. 11) are in a somewhat better agreement with the experiment.

The resonant energies of the incident electron as well as the energies and the widths of the corresponding autoionizing states for helium-like boron are presented in Table $\mathrm{V}$ and compared with data taken from [5]. A good agreement of our results with the theoretical and experimental data of [5] shows that our approach can also be applied to relatively light systems.

It is worth noting that experimental data for elastic resonant electron scattering are available only for rather light ions: $\mathrm{B}^{4+}, \mathrm{C}^{5+}, \mathrm{N}^{+6}, \mathrm{O}^{7+}$, and $\mathrm{F}^{8+}$ [5,25]. Consequently, the considered impact energies were relatively modest and such scattering processes could be successfully described even by a nonrelativistic theory. To our knowledge, experiments on electron resonant scattering on ions with a (much) larger charge are still absent. However, recently such experiments have become feasible [26]. The most appropriate means for measuring the cross sections of resonant electron scattering on heavy highly charged ions are storage rings of heavy ions. Two types of targets can be employed: a beam of free electrons or a gas of light atoms which serve as a source of quasifree electrons. For instance, the former experimental setup was used to measure the cross section for dielectronic recombination with $\mathrm{H}$-like uranium [27]. For registration of scattered electrons, an electron detector with a fine energy resolution is necessary. Detectors with the required resolution are available at the GSI [28,29] and also at some other scientific centers [30,31]. Hence, all the ingredients to perform experiment on resonant electron scattering on heavy highly charged ions are already available and the relativistic QED theory of this process, presented in our paper, may serve as a guidance for experimental studies.

Finally, in this section we would like to note the following. As was already mentioned in the Introduction, the resonant part of electron scattering on a hydrogen-like uranium ion was calculated in [9]. We also considered electron scattering on hydrogen-like uranium taking into account not only the resonant part, but also the Coulomb scattering and the interference between them. In this case, the Coulomb scattering turned out to be by far the dominant one. However, our results obtained by neglecting the Coulomb scattering (and the interference) are in a good agreement with those of Kollmar et al. [9].

\section{CONCLUSION}

We have considered elastic scattering of an electron on a hydrogen-like highly charged ion. The focus of the study has been on electron impact energies where autoionizing states of the corresponding helium-like ion may play an important role in the process. Compared to electron scattering on light ions, the main difference in the present case is a strong field generated by the nucleus of the highly charged ions which makes it necessary to take into account the relativistic and QED effects. To this end, we have developed $a b$ initio relativistic QED theory for elastic electron scattering on hydrogen-like highly charged ions which describes in a unified and self-consistent way both the direct (Coulomb) scattering and resonant scattering proceeding via formation and consequent decay of autoionizing states.

Using this theory we have calculated scattering cross sections for a number of collision systems ranging from relatively light to very heavy ones. As one could expect, with increasing the charge of the ionic nucleus, the role of the resonant scattering decreases. However, even for ions with $Z \approx 50$ the resonances in the cross section remain clearly visible for backward scattering.

Although the presented theory has been developed first of all for the description of collisions with highly charged ions, its application for such a light system as $\left(e^{-}+\mathrm{B}^{4+}\right)$ demonstrates that it can be successfully used for an accurate description of a very broad range of colliding systems.

\section{ACKNOWLEDGMENTS}

The authors are grateful to D. Yu, S. Hagmann, and P.-M. Hillenbrand for helpful discussions. The work of D.M.V. and O.Y.A. on the calculation of the differential cross sections was supported solely by the Russian Science Foundation under Grant No. 17-12-01035. The work of O.Y.A. was partly supported by Ministry of Education and Science of the Russian Federation under Grant No. 3.1463.2017/4.6. A.B.V. acknowledges the support from the Deutsche Forschungsgemeinschaft (DFG, German Research Foundation) under Grant No 349581371 (VO 1278/4-1).

\section{APPENDIX A: COULOMB SCATTERING}

The Coulomb scattering amplitude can be calculated as the scalar product of the in and out states $\left(\psi^{(-)}\right.$and $\left.\psi^{(+)}\right)$

$$
S_{i f}=\left\langle\psi_{\boldsymbol{p}_{f} \mu_{f}}^{(-)} \mid \psi_{\boldsymbol{p}_{i} \mu_{i}}^{(+)}\right\rangle=\int d^{3} \boldsymbol{r} \psi_{\boldsymbol{p}_{f} \mu_{f}}^{(-)+}(\boldsymbol{r}) \psi_{\boldsymbol{p}_{i} \mu_{i}}^{(+)}(\boldsymbol{r}) .
$$

We assume that the $z$ axis is directed along the electron momentum $\boldsymbol{p}_{i}$ in the initial state. Presenting the in and out 
states in the form given by Eq. (3), the $S$-matrix element reads as

$$
\begin{aligned}
S_{i f}= & N^{2} \sum_{j l m} \sum_{j^{\prime} l^{\prime} m^{\prime}} M_{j^{\prime} l^{\prime} m^{\prime} \mu_{f}}^{*}(\theta, \varphi) M_{j l m \mu_{i}}(0,0) e^{i\left(\phi_{j l}+\phi_{j^{\prime} l^{\prime}}\right)} i^{l-l^{\prime}} \\
& \times \int d^{3} \boldsymbol{r} \psi_{\varepsilon^{\prime} j^{\prime} l^{\prime} m^{\prime}}^{+}(\boldsymbol{r}) \psi_{\varepsilon j l m}(\boldsymbol{r}) \\
= & \left.N^{2} \delta\left(\varepsilon-\varepsilon^{\prime}\right) \sum_{j l m} M_{j l m \mu_{f}}^{*}(\theta, \varphi) M_{j l m \mu_{i}}(0,0) e^{2 i \phi_{j l}}, \quad \text { (A2 }\right)
\end{aligned}
$$

where we introduced

$$
\begin{aligned}
M_{j l m \mu}(\boldsymbol{v}) & =\Omega_{j l m}^{+}(\boldsymbol{v}) v_{\mu}(\boldsymbol{v}) \\
& =\sum_{m_{l} m_{s}} C_{j m}^{l s}\left(m_{l}, m_{s}\right) Y_{l m_{l}}^{*}(\theta, \varphi) \chi_{m_{s}}^{+} v_{\mu}(\theta, \varphi) \\
& =\sum_{m_{l} m_{s}} C_{j m}^{l s}\left(m_{l}, m_{s}\right) Y_{l m_{l}}^{*}(\theta, 0) \chi_{m_{s}}^{+} v_{\mu}(\theta, \varphi) e^{i\left(m_{s}-m\right) \varphi} .
\end{aligned}
$$

Taking into account that

$$
\begin{gathered}
Y_{l m_{l}}(0,0)=\sqrt{\frac{2 l+1}{4 \pi}} \delta_{m_{l} 0}, \\
\chi_{m_{s}}^{+} v_{\mu}(0,0)=\delta_{\mu m_{s}},
\end{gathered}
$$

we obtain

$$
M_{j l m \mu}(0,0)=C_{j m}^{l s}(0, \mu) \sqrt{\frac{2 l+1}{4 \pi}} \sim \delta_{m \mu} .
$$

It is convenient to introduce the quantity

$$
\left(v_{\mu}\right)_{m_{s}}=\chi_{m_{s}}^{+} v_{\mu}(\theta, \varphi)
$$

Then, the $S$-matrix element can be written as

$$
\begin{aligned}
S_{i f} & =N^{2} \delta\left(\varepsilon-\varepsilon^{\prime}\right) \sum_{j l} \sum_{m_{l} m_{s}} C_{j \mu_{i}}^{l s}\left(m_{l}, m_{s}\right) Y_{l m_{l}}(\theta, 0)\left[\chi_{m_{s}}^{+} v_{\mu_{f}}(\theta, \varphi)\right]^{*} e^{i\left(\mu_{i}-m_{s}\right) \varphi} C_{j \mu_{i}}^{l s}\left(0, \mu_{i}\right) \sqrt{\frac{2 l+1}{4 \pi}} e^{2 i \phi_{j l}} \\
& =(-2 \pi i) \delta\left(\varepsilon-\varepsilon^{\prime}\right) N^{2} \frac{i}{2 \pi} \sum_{m}\left(v_{\mu_{f}}\right)_{m}^{*} e^{i\left(\mu_{i}-m\right) \varphi} \sum_{j l} \sqrt{\frac{2 l+1}{4 \pi}} C_{j \mu_{i}}^{l s}\left(\mu_{i}-m, m\right) C_{j \mu_{i}}^{l s}\left(0, \mu_{i}\right) e^{2 i \phi_{j l}} Y_{l \mu_{i}-m}(\theta, 0) .
\end{aligned}
$$

Making use of Eq. (18) we get

$$
R_{i f}^{\text {Coul }}=N^{2} \frac{(-1) p}{(2 \pi)^{2}} \sum_{m}\left(v_{\mu_{f}}\right)_{m}^{*} M_{m \mu_{i}}
$$

where, following [17], we introduced the matrix $M_{m \mu_{i}}$ :

$$
M_{m \mu_{i}}=4 \pi \frac{1}{2 p i} e^{i\left(\mu_{i}-m\right) \varphi} \sum_{j l} \sqrt{\frac{2 l+1}{4 \pi}} C_{j \mu_{i}}^{l s}\left(\mu_{i}-m, m\right) C_{j \mu_{i}}^{l s}\left(0, \mu_{i}\right) Y_{l \mu_{i}-m}(\theta, 0) e^{2 i \phi_{j l}} .
$$

In particular,

$$
\begin{gathered}
M_{m \mu}=4 \pi \frac{1}{2 p i} \sum_{j l} \sqrt{\frac{2 l+1}{4 \pi}} C_{j \mu}^{l s}(0, \mu) C_{j \mu}^{l s}(0, \mu) Y_{l 0}(\theta, 0) e^{2 i \phi_{j l},}, \quad \text { if } m=\mu \\
M_{m \mu}=4 \pi \frac{1}{2 p i} e^{2 i \mu \varphi} \sum_{j l} \sqrt{\frac{2 l+1}{4 \pi}} C_{j \mu}^{l s}(2 \mu, \bar{\mu}) C_{j \mu}^{l s}(0, \mu) Y_{l 2 \mu}(\theta, 0) e^{2 i \phi_{j l}}, \quad \text { if } m=-\mu .
\end{gathered}
$$

Accordingly, we obtain

$$
\begin{aligned}
& M_{+1 / 2,+1 / 2}(\theta, \varphi)=f(\theta), \quad M_{+1 / 2,-1 / 2}(\theta, \varphi)=g(\theta) e^{-i \varphi} \\
& M_{-1 / 2,+1 / 2}(\theta, \varphi)=-g(\theta) e^{i \varphi}, \quad M_{-1 / 2,-1 / 2}(\theta, \varphi)=f(\theta) .
\end{aligned}
$$

Taking into account that

$$
\begin{gathered}
Y_{l 1}(\theta, 0)=-Y_{l,-1}(\theta, 0)=-\sqrt{\frac{(2 l+1)(l-1) !}{4 \pi(l+1) !}} P_{l}^{1}(\cos \theta), \\
Y_{l 0}(\theta, 0)=\sqrt{\frac{2 l+1}{4 \pi}} P_{l}(\cos \theta), \\
\left(C_{j m}^{l 1 / 2}(0, m)\right)^{2}=\frac{|\varkappa|}{2 l+1},
\end{gathered}
$$




$$
\begin{array}{cc}
C_{j m}^{l s}(2 m, \bar{m}) C_{j m}^{l s}(0, m)=\frac{1}{2 l+1} \sqrt{l(l+1)}, \quad j=l+\frac{1}{2} \\
C_{j m}^{l s}(2 m, \bar{m}) C_{j m}^{l s}(0, m)=-\frac{1}{2 l+1} \sqrt{l(l+1)}, \quad j=l-\frac{1}{2}
\end{array}
$$

we obtain

$$
\begin{gathered}
f(\theta)=\frac{1}{2 p i} \sum_{j l}|\varkappa|\left(e^{2 i \phi_{\varkappa}}-1\right) P_{l}(\cos \theta), \\
g(\theta)=\frac{1}{2 p i} \sum_{l}\left(e^{2 i \phi_{\varkappa=-l-1}}-e^{2 i \phi_{\varkappa=l}}\right) P_{l}^{1}(\cos \theta),
\end{gathered}
$$

where $\phi_{\varkappa} \equiv \phi_{j l}$ and $\varkappa$ is the Dirac quantum number

$$
\varkappa=\left(j+\frac{1}{2}\right)(-1)^{j+l+1 / 2} .
$$

Using Eq. (69), the differential cross section for the Coulomb scattering is obtained to be

$$
\begin{gathered}
d \sigma_{i f}=2 \pi\left|R_{i f}\right|^{2} \frac{\varepsilon}{p} \delta\left(E_{i}-E_{f}\right) \frac{d^{3} \boldsymbol{p}}{(2 \pi)^{3}}, \\
\frac{d \sigma_{i f}}{d \boldsymbol{v}}=\left|\sum_{m}\left(v_{\mu_{f}}\right)_{m}^{*} M_{m \mu_{i}}\right|^{2} .
\end{gathered}
$$

\section{APPENDIX B: NUMERICAL CALCULATION OF THE COULOMB AMPLITUDES}

The Coulomb amplitudes are given by series equations (A19) and (A20). These series are not convenient for a direct numerical calculation. However, the leading part of these series can be calculated analytically and the remaining part can be easily summed up numerically.

The Coulomb phase shifts for the potential $V=-\alpha Z / r$ read as

$$
\phi_{\varkappa}=\arg \Gamma(\gamma-i \nu)+\eta-\frac{1}{2} \pi \gamma+\frac{\pi}{2}(l+1),
$$

where

$$
\begin{gathered}
e^{2 i \eta}=\frac{-\varkappa+i v^{\prime}}{\gamma+i v}, \\
v=\frac{\varepsilon}{p} \alpha Z, \\
v^{\prime}=\frac{m_{e}}{p} \alpha Z, \\
\gamma=\sqrt{\varkappa^{2}-\alpha^{2} Z^{2}},
\end{gathered}
$$

where $\alpha$ is the fine-structure constant and $m_{e}$ is the electron mass. Following [32] we introduce

$$
\begin{aligned}
a_{\varkappa}(\gamma) & =e^{2 i \phi_{\varkappa}}-1 \\
& =(-1)^{l+1}\left(\frac{-\varkappa+i \nu^{\prime}}{\gamma+i \nu}\right) \frac{\Gamma(\gamma-i \nu)}{\Gamma(\gamma+i \nu)} e^{-i \pi \gamma}-1 .
\end{aligned}
$$

Then, the amplitudes (A19) and (A20) can be written as

$$
\begin{aligned}
f(\theta) & =\frac{1}{2 i p} \sum_{j l}|\varkappa| a_{\varkappa} P_{l}(\cos \theta) \\
& =\frac{1}{2 i p} \sum_{l}\left[(l+1) a_{\varkappa=-l-1}+l a_{\varkappa=l}\right] P_{l}(\cos \theta), \\
& g(\theta)=\frac{1}{2 i p} \sum_{l}\left(a_{\varkappa=-l-1}-a_{\varkappa=l}\right) P_{l}^{1}(\cos \theta) .
\end{aligned}
$$

Now, we introduce the approximate amplitudes

$$
\begin{gathered}
\tilde{f}(\theta)=\frac{1}{2 i p} \sum_{l}\left[(l+1) \tilde{a}_{\varkappa=-l-1}+l \tilde{a}_{\varkappa=l}\right] P_{l}(\cos \theta), \\
\tilde{g}(\theta)=\frac{1}{2 i p} \sum_{l}\left[\tilde{a}_{\varkappa=-l-1}-\tilde{a}_{\varkappa=l}\right] P_{l}^{1}(\cos \theta),
\end{gathered}
$$

where

$$
\tilde{a}_{\varkappa}=a_{\varkappa}(\gamma=|\varkappa|)
$$

and, correspondingly,

$$
\begin{gathered}
\tilde{a}_{\varkappa=-l-1}=\left(l+1+i v^{\prime}\right) \frac{\Gamma(l+1-i v)}{\Gamma(l+2+i v)}-1, \\
\tilde{a}_{\varkappa=l}=\left(l-i v^{\prime}\right) \frac{\Gamma(l-i v)}{\Gamma(l+1+i v)}-1 .
\end{gathered}
$$

The series equations (B9) and (B10) can be summed up analytically [32]:

$$
\begin{gathered}
\tilde{f}(\theta)=\frac{\Gamma(1-i v)}{\Gamma(1+i v)} e^{i v \ln \sin ^{2}(\theta / 2)}\left[\frac{v}{2 p} \csc ^{2}(\theta / 2)+\frac{v^{\prime}-v}{2 p}\right], \\
\tilde{g}(\theta)=\frac{\Gamma(1-i v)}{\Gamma(1+i v)} e^{i v \ln \sin ^{2}(\theta / 2)}\left[\frac{v^{\prime}-v}{2 p} \cot (\theta / 2)\right] .
\end{gathered}
$$

As a result, the Coulomb amplitudes can be rewritten as

$$
\begin{aligned}
f(\theta)= & \tilde{f}(\theta)+\frac{1}{2 i p} \sum_{j l}|\varkappa|\left(a_{\varkappa}-\tilde{a}_{\varkappa}\right) P_{l}(\cos \theta) \\
= & \tilde{f}(\theta)+\frac{1}{2 i p} \sum_{l}\left[(l+1)\left(a_{\varkappa=-l-1}-\tilde{a}_{\varkappa=-l-1}\right)\right. \\
& \left.+l\left(a_{\varkappa=l}-\tilde{a}_{\varkappa=l}\right)\right] P_{l}(\cos \theta), \\
g(\theta)= & \tilde{g}(\theta)+\frac{1}{2 i p} \sum_{l}\left(a_{\varkappa=-l-1}-\tilde{a}_{\varkappa=-l-1}-a_{\varkappa=l}\right. \\
& \left.+\tilde{a}_{\varkappa=l}\right) P_{l}^{1}(\cos \theta),
\end{aligned}
$$

where the corresponding series can be easily calculated numerically. 
[1] B. A. Huber, C. Ristori, C. Guet, D. Küchler, and W. R. Johnson, Phys. Rev. Lett. 73, 2301 (1994).

[2] J. B. Greenwood, I. D. Williams, and P. McGuinness, Phys. Rev. Lett. 75, 1062 (1995).

[3] G. Toth, S. Grabbe, P. Richard, and C. P. Bhalla, Phys. Rev. A 54, R4613 (1996).

[4] T. J. M. Zouros, E. P. Benis, and T. W. Gorczyca, Phys. Rev. A 68, 010701(R) (2003).

[5] E. P. Benis, T. J. M. Zouros, T. W. Gorczyca, A. D. González, and P. Richard, Phys. Rev. A 69, 052718 (2004).

[6] E. P. Benis, T. J. M. Zouros, T. W. Gorczyca, A. D. González, and P. Richard, Phys. Rev. A 73, 029901(E) (2006).

[7] D. C. Griffin and M. S. Pindzola, Phys. Rev. A 42, 248 (1990).

[8] C. P. Bhalla, Phys. Rev. Lett. 64, 1103 (1990).

[9] K. Kollmar, N. Grün, and W. Scheid, Eur. Phys. J. D 10, 27 (2000).

[10] V. A. Zaytsev, S. Tashenov, A. V. Maiorova, V. M. Shabaev, and T. Sthlker, J. Phys. B: At., Mol. Opt. Phys. 48, 165003 (2015).

[11] W. H. Furry, Phys. Rev. 81, 115 (1951).

[12] A. I. Akhiezer and V. B. Berestetskii, Quantum Electrodynamics (Wiley Interscience, New York, 1965).

[13] D. A. Varshalovich, A. N. Moskalev, and V. K. Khersonskii, Quantum Theory of Angular Momentum (World Scientific, Singapore, 1988).

[14] O. Y. Andreev, L. N. Labzowsky, G. Plunien, and D. A. Solovyev, Phys. Rep. 455, 135 (2008).

[15] M. Gell-Mann and M. L. Goldberger, Phys. Rev. 91, 398 (1953).

[16] B. A. Lippmann and J. Schwinger, Phys. Rev. 79, 469 (1950).
[17] P. Burke, R-matrix Theory of Atomic Collisions (Springer, Berlin, 2011).

[18] M. Gell-Mann and F. Low, Phys. Rev. 84, 350 (1951).

[19] J. Sucher, Phys. Rev. 107, 1448 (1957).

[20] L. N. Labzovskii, Zh. Eksp. Teor. Fiz. 59, 168 (1970) [JETP 32, 94 (1971)].

[21] V. Shabaev, Phys. Rep. 356, 119 (2002).

[22] I. Lindgren, S. Salomonson, and B. Sn, Phys. Rep. 389, 161 (2004).

[23] F. Low, Phys. Rev. 88, 53 (1952).

[24] O. Y. Andreev, L. N. Labzowsky, and A. V. Prigorovsky, Phys. Rev. A 80, 042514 (2009).

[25] G. Toth, P. Zavodszky, C. P. Bhalla, P. Richard, S. Grabbe, and H. Aliabadi, Phys. Scr. T92, 272 (2001).

[26] D. Yu, S. Hagmann and P.-M. Hillenbrandt (private communication).

[27] D. Bernhardt, C. Brandau, Z. Harman, C. Kozhuharov, A. Müller, W. Scheid, S. Schippers, E. W. Schmidt, D. Yu, A. N. Artemyev et al., Phys. Rev. A 83, 020701(R) (2011).

[28] P.-M. Hillenbrand, S. Hagmann, Y. A. Litvinov, and T. Sthlker, J. Phys.: Conf. Ser. 635, 012011 (2015).

[29] P.-M. Hillenbrand, S. Hagmann, A. B. Voitkiv, B. Najjari, D. Banaś, K.-H. Blumenhagen, C. Brandau, W. Chen, E. De Filippo, A. Gumberidze et al., Phys. Rev. A 90, 042713 (2014).

[30] W. Mampe, K. Schreckenbach, P. Jeuch, B. Maier, F. Braumandl, J. Larysz, and T. von Egidy, Nucl. Instrum. Methods 154, 127 (1978).

[31] D. Varga, I. Kádár, S. Ricz, J. Végh, A. Kövér, B. Sulik, and D. Berényi, Nucl. Instrum. Methods Phys. Res., Sect. A 313, 163 (1992).

[32] W. R. Johnson, Approximate Coulomb scattering amplitudes, https://www3.nd.edu/ johnson/Publications/scatter.pdf. 